\title{
Hedgehog Signaling Pathway Regulates Autophagy in Human Hepatocellular Carcinoma Cells
}

\author{
Ying Wang ${ }^{1,2}$, Chang Han ${ }^{1}$, Lu Lu1 ${ }^{1}$, Susan Magliato1, and Tong $\mathbf{W u}^{1}$ \\ ${ }^{1}$ Department of Pathology and Laboratory Medicine, Tulane University School of Medicine, 1430 \\ Tulane Avenue SL-79, New Orleans, Louisiana 70112 \\ ${ }^{2}$ Department of Gastroenterology and Internal Medicine, Tongji Hospital, Tongji Medical College, \\ Huazhong University of Science and Technology, Wuhan, China
}

\section{Abstract}

Hedgehog (Hh) signaling plays an important role in embryonic development and in the regulation of a variety of cellular functions. Aberrant activation of Hh signaling has been implicated in several human cancers including hepatocellular carcinoma (HCC). In this study we examined the pathobiological functions and molecular mechanisms of Hh signaling pathway in HCC cells. Treatment of cultured human HCC cells (Huh7, Hep3B and HepG2) with the Hh signaling ligand (recombinant Shh) or agonist, SAG and purmorphamine, prevented the induction of autophagy. In contrast, GANT61 (a small molecule inhibitor of Gli1 and Gli2) induced autophagy, as determined by immunobloting for microtubule-associated protein light chain 3 (LC3) and p62, GFP-LC3 puncta, monodansylcadaverine (MDC) staining and transmission electron microscopy. Hh inhibition-induced autophagy was associated with upregulation of Bnip3, as determined by immunoblotting and real-time PCR assay. Knockdown of Bnip3 by RNAi impaired GANT61induced autophagy. Additionally, Hh inhibition-induced autophagy was associated with Bnip3mediated displacement of Bcl-2 from Beclin-1, as determined by immunoblotting and immunoprecipitation assays. Furthermore, inhibition of Hh signaling increased HCC cell apoptosis and decreased cell viability, as determined by caspase and WST-1 assays. Pharmacological or genetic inhibition of autophagy by 3-methyladenine (3-MA) or Beclin-1 siRNA partially suppressed GANT61-induced cell apoptosis and cytotoxicity. In a tumor xenograft model using SCID mice inoculated with Huh7 cells, administration of GANT61 inhibited tumor formation and decreased tumor volume; this effect was partially blocked by the autophagy inhibitor, 3-MA. These findings provide novel evidence that hedgehog inhibition induces autophagy through upregulation of Bnip3 and that this mechanism contributes to apoptosis. Therefore, the status of autophagy is a key factor that determines the therapeutic response to Hh-targeted therapies.

\section{Keywords}

Hedgehog signaling; GANT61; autophagy; hepatocellular carcinoma; apoptosis

\footnotetext{
Address correspondence to: Tong Wu, M.D., Ph.D., Department of Pathology and Laboratory Medicine, Tulane University School of Medicine, 1430 Tulane Avenue, SL-79, New Orleans, LA 70112, Tel: 504-988-5210, Fax: 504-988-7862, twu@ tulane.edu.

No conflicts of interest exist
} 


\section{INTRODUCTION}

Hedgehog (Hh) was initially discovered nearly 30 years ago as a "segmentpolarity" gene that controls Drosophila embryonic cuticle pattern(1). Since the discovery of its vertebrate counterparts in the early 1990s, enormous progress has been made in revealing the role of $\mathrm{Hh}$ signaling in development and disease as well as the molecular underpinning of the $\mathrm{Hh}$ signaling cascade(2). We now know that Hh signaling plays an important role in embryonic development and in the regulation of a variety of cellular functions including proliferation, survival, stemness and differentiation. The Hh signaling pathway consists of Hh ligands (Sonic Hh, Indian Hh, and Desert Hh), the 12-span transmembrane protein, Patched (Ptc), as the Hh receptor, the 7-span transmembrane protein, Smoothened (Smo), as the obligatory signal transducer across the plasma membrane, and the 5-zinc finger transcription factor Glis (Gli-1, Gli-2, and Gli-3)(3). Activation of the canonical Hh signaling pathway is initiated by the binding of Hh ligands to their receptor, Ptc, which becomes internalized leading to the activation of Smo via release from Ptc-dependent suppression. Smo activates the final arbiter of Hh signaling, the Gli family of transcription factors that regulate Hh target genes expression, including Ptch1 and Gli1. Recently, aberrant activation of Hh signaling has been implicated in several human malignancies including hepatocellular carcinoma $(\mathrm{HCC})(4,5)$.

HCC is one of the most common malignancies and the leading causes of cancer related mortality(6). The overall survival of patients with HCC has not significantly improved in the past two decades. Current treatments are only applicable at early stages of tumor development, including surgery and chemotherapy. But a majority of patients has an advanced or unresectable disease at presentation which makes the prognosis of HCC dismal. Conventional systemic chemotherapy options have typically yielded poor outcomes for these patients. Although in recent years several clinical trials have tested the efficacy of agents that selectively target important signaling pathways involved in the control of HCC, no relevant improvement in patient prognosis has been achieved so far. Therefore, it is urgent and practical to identify novel therapeutic strategies for more effective therapy. In this context, it is encouraging that Hhtargeted therapy has emerged as a potential new treatment for Hh-dependent human cancers including $\mathrm{HCC}(7)$.

Autophagy is an evolutionarily conserved process that involves lysosomal degradation of cytoplasmic and cellular organelles, which consists of several steps including sequestration, transport to lysosomes, degradation, and utilization of degradation products(8). It is characterized by progressive formation of vesicle structures from autophagosomes to autophagolysosomes, orchestrated by autophagy effectors (Atg proteins) and modulators (i.e., class III phosphatidylinositol-3-kinase). The process of autophagosome formation involves two major steps: nucleation and elongation of the isolation membrane. The Atg1/ unc-51-like kinase (ULK) complex, the autophagy-specific PI3-kinase complex, and PI3Peffectors and their related proteins are important for the nucleation step, whereas the Atg12and LC3/Atg8-conjugation systems are important for the elongation step. Studies have demonstrated that autophagy plays a wide variety of physiological and pathophysiological roles. Recent evidence has shown that autophagy is associated with cancer pathogenesis and that pharmacologic manipulation of autophagic pathways may represent a new therapeutic strategy for human cancers. However, to date, the role of autophagy in cancer is not clearly defined. Although autophagy is a cancer cell survival mechanism against environmental and cellular stresses, it can be associated with cancer cell death under certain situations. Further, autophagy and apoptosis might be linked to each other and occur simultaneously or sequentially in a cell type-, death stimulus-, and context-dependent manner(9).

Although Hh signaling is known to inhibit cell apoptosis, it remains unknown whether Hh signaling is able to regulate autophagy. The current study describes a novel role of $\mathrm{Hh}$ 
signaling pathway for regulation of autophagy in human HCC cells. We show that inhibition of Hh pathway markedly enhanced autophagy through up-regulation of Bnip3 (a member of BH3- only subset of the Bcl-2 family) that displaces Bcl-2 from its binding partner, Beclin-1, and that this process preludes apoptosis. Our findings suggest that the status of autophagy (autophagic flux) is a key factor that may influence cell response to Hh-targeted therapy.

\section{EXPERIMENTAL PROCEDURES}

Human HCC cells (Huh7, Hep3B and HepG2) were treated with the Hh signaling ligand, agonists or inhibitors as indicated and the cells were analyzed for autophagy by immunobloting for microtubule-associated protein light chain 3 (LC3) and p62, GFP-LC3 puncta, monodansylcadaverine (MDC) staining and transmission electron microscopy. Western blotting analysis was performed to determine the alteration of key signaling molecules including Shh, Patched, Smo, and Gli1, Bnip3, Bcl-2 family proteins and MEK/ ERK1/2.

The interaction between Bcl-2 and Beclin-1 was analyzed by immunoprecipitation and immunoblotting assays.

For qRT-PCR, total RNA was extracted with TRIzol ${ }^{\circledR}$ plus RNA purification kit and reverse-transcribed to cDNA using Superscript ${ }^{\mathrm{TM}}$ II reverse transcriptase; the cDNA samples were used for real-time PCR analysis in triplicate using QuantiFast SYBR Green PCR kit (Qiagen) on Bio-Rad C1000 Thermal Cycler.

For plasmid or siRNA transfection, the Bcl-2 and Bnip3 expression plasmid or Bnip3 siRNA were transfected into Huh7 cells by using Lipofectamine 2000; 6 h post-transfection, the cells were treated with indicated reagents and analyzed for apoptosis while the cell lysates were obtained for Western blotting and immunoprecipitation.

For flow cytometry analysis of apoptosis, the cells were harvested, centrifuged and resuspended in $100 \mu \mathrm{l}$ Annexin-V-FLUOS labeling solution containing of $2 \mu \mathrm{l}$ Annexin-VFLUOS labeling reagent and $2 \mu \mathrm{l}$ Propidium iodide solution and the cells were analyzed on a FACScan Flow Cytometer (BD LSRII).

A tumor xenograft model was utilized to evaluate the effect of Hh inhibition on HCC growth in SCID mice. Male SCID mice were subcutaneously inoculated into flank with $1 \times 10^{7}$ Huh7 cells. One week post-inoculation, the mice were randomized to three groups, and treated with vehicle only, GANT61 $(50 \mathrm{mg} / \mathrm{kg})$ and GANT61 $(50 \mathrm{mg} / \mathrm{kg})$ combination with 3-MA $(10 \mathrm{mg} / \mathrm{kg}$ ) by intraperitoneal (i.p.) injection every other day for 4 weeks. The animals were closely observed to document the tumor growth parameters. The tumor tissues were utilized for H\&E staining, western blotting analysis for LC3II and caspases, and immunofluorescent staining for LC3II.

\section{RESULTS}

\section{Hh Signaling Pathway is active in human HCC cells}

Western blotting analysis showed that canonical Hh signaling pathway components, including the ligand, Shh, and the signaling molecules, Patched, Smo, and Gli1, were expressed in HCC cell lines (Huh7, HepG2 and Hep3B) (Figure 1A). These observations are consistent with the reported upregulation of Hh pathway components in HCC cells and tissues $(4,5)$. To determine Hh signaling activity in these cells, we employed a Glidependent luciferase reporter system(2), in which the cells were transfected with a Gli- 
dependent luciferase reporter construct, followed by treatment with recombinant Shh (a $\mathrm{Hh}$ ligand), SAG (a Hh agonist that acts downstream by directly binding to Smoothened), purmorphamine (a Hh agonist directly targeting Smoothened), GDC-0449 (Smoothened antagonist), or GANT61 (a small molecule inhibitor of Gli1 and Gli2), respectively. As shown in Figure 1B, activation of Hh signaling by its ligand (Shh) and agonists (SAG or Pur) enhanced Gli-dependent luciferase reporter activity, whereas inhibition of Hh signaling by GANT61 and GDC-0449 reduced Gli reporter activity. Accordingly, activation of $\mathrm{Hh}$ signaling by Shh, SAG or Pur in Huh7 cells increased the mRNA levels of two Gli target genes, Ptch1 and Gli1, while inhibition of Hh signaling by GANT61 or GDC-0449 reduced Ptch1 and Gli1 mRNAs (Figure 1C). The Gli inhibitor, GANT61, reduced Gli reporter activity and downstream gene expression to a greater extent than the Smo inhibitor, GDC-0449. These findings suggest an autocrine mode of Hh signaling activation in HCC cells.

\section{Inhibition of Hh signaling induces autophagy}

To determine the impact of Hh signaling on autophagy, Huh7, HepG2 and Hep3B cells were treated with the Hh pathway agonists or antagonists and the cells were analyzed to determine the level of LC3II, an essential component and a widely used marker for autophagosomes. Western blotting analysis showed that treatment with the Gli inhibitor, GANT61, induced the accumulation of LC3II in all three HCC cell lines (Figure 2A). Treatment with the Smo inhibitor, GDC-0449, also increased LC3II level, albeit the effect was less prominent compared to GANT61. In contrast, activation of Hh signaling by its ligand (Shh) and agonists (SAG or Pur) decreased the level of LC3II.

In addition to LC3II western blotting, we further utilized fluorescence microscopy to determine the redistribution of GFP-LC3 (LC3 is a mammalian homolog of yeast Atg8 and is normally expressed in a diffuse pattern in resting cells; during autophagy, autophagosomes engulf bulk cytoplasmic constituents including proteins and organelles, and along this process, the cytosolic form of LC3 [LC3I] is conjugated to phosphatidylethanolamine to form LC3II, which is recruited to autophagosomal membranes resulting in a more punctate distribution pattern). As shown in Figure 2B, GANT61 treatment induced GFP-LC3 dot redistribution from a diffuse pattern to a punctate cytoplasmic pattern (GFP-LC3 puncta) in all three HCC cell lines. The Smo inhibitor, GDC-0449, also induced GFP-LC3 puncta formation, although the effect was slightly less prominent compared to GANT61. These findings indicate that inhibition of Hh signaling induces autophagy and that the Gli inhibitor, GANT61, is a potent agent that induces autophagy. Although GANT61-induced autophagy is observed in all three HCC cell lines, the effect is most prominent in Huh7 cells (Figure 2A).

To further document the effect of GANT61 on autophagy, we performed dose-dependent experiments in Huh7 cells (the cells were treated with GANT61 at $5 \mu \mathrm{M}, 10 \mu \mathrm{M}$ or $20 \mu \mathrm{M}$ concentration for 24 and 48 hours; quantitative assessment for the ratio of LC3II to LC3I was used as the primary indicator of autophagy induction). As shown in Figure 2C, GANT61-induced LC3II accumulation in a dose-dependent manner.

Increased detection of autophagic markers, such as LC3II accumulation and GFP-LC3 redistribution, can result from either increased autophagosome formation or inhibition of ongoing autophagosomal maturation(10). To delineate these possibilities, the cells were pretreated with 3-methyladenine (3-MA, a classical inhibitor of autophagy at the sequestration stage) or E-64d/pepstatin A (lysosomal protease inhibitors that block autophagolysosomal degradation) prior to GANT61 treatment. As shown in Figure 2D, 3MA treatment abolished GANT61-induced LC3-II formation, whereas E-64d/pepstatin A treatment augmented GANT61-induced LC3-II accumulation. The protein, p62/SQSTM1, 
binds directly to LC3, incorporates into the completed autophagosomes, and becomes degraded in autolysosomes. In our system we observed that GANT61 treatment decreased the level of p62 in Huh7 cells and the effect was reversed by 3-MA and E-64d/pepstatin A. Taken together, these findings suggest that the Gli inhibitor, GANT61, enhanced autophagic flux.

In addition to utilization of LC3II accumulation and GFP-LC3 redistribution as markers of autophagy, we further employed MDC (monodansylcadaverine) staining and transmission electron microscopy to verify induction of autophagy by GANT61. MDC is an autofluorescent agent that is accumulated specifically in autophagolysosomes. As shown in Supplemental Figure S1A, treatment with GANT61 and GDC-0449 induced the accumulation of MDC in the cytoplasmic vacuoles in Huh7 cells (the accumulation was greater in GANT61-treated cells compared to GDC-0449-treated cells). Transmission electron microscopy also showed formation of autophagosomes and autophagolysosomes in GANT61 treated Huh7 cells, characterized by double-membrane vacuolar structures containing cytoplasmic contents (Supplemental Figure S1B).

\section{Activation of Hh signaling prevents autophagy}

To assess the impact of Hh signaling activation on autophagy, HCC cells were treated with autophagy-inducing drugs (carbamazepine and oxaliplatin) in the presence or absence of $\mathrm{Hh}$ ligand (Shh) or agonists (SAG or Pur) (carbamazepine is an autophagy-enhancing drug for hepatocytes; oxaliplatin is a second generation potent platinum-based anti-neoplastic agent that can induce autophagy in HCC cells). As shown in Figure 3A and 3B, activation of $\mathrm{Hh}$ signaling by Shh, SAG or Pur prevented carbamazepine and oxaliplatin-induced LC3II accumulation in all three HCC cells; these findings indicate that activation of Hh signaling is able to prevent autophagy in HCC cells. In contrast, inhibition of Hh pathway by GDC0449 or GANT61 enhanced carbamazepine and oxaliplatin-induced LC3II accumulation in all three HCC cells, which suggest that inhibition of Hh signaling synergizes with autophagyinducing drugs in autophagy induction (Figure 3C and 3D).

\section{GANT61 does not increase ATG gene expression}

ATG (autophagy-related) genes encode proteins required for autophagy and play essential roles in autophagy. Autophagosome formation is mediated by two ubiquitin-like conjugation systems composed of Atg proteins, which culminate in conjugation of Atg12 to Atg5 and conversion of a soluble form of LC3-I to phosphatidylethanolamine-conjugated membranebound form (LC3-II)(8). The proteins Atg3, Atg5, Atg6/Beclin1, Atg7, and Atg12 are involved in autophagosome formation and are well conserved from yeast to humans. Because many autophagic triggers upregulate ATG genes, we examined whether GANT61 treatment might influence the expression levels of ATG genes in HCC cells. As shown in Supplemental Figure S2, GANT61 treatment did not increase the expression of ATG genes (Atg3 levels was slightly decreased in GANT61-treated Huh7 and Hep3B cells compared to cells treated with vehicle or Hh ligand/agonists). These results suggest GANT61-induced autophagy is not associated with up-regulation of ATG gene expression.

\section{Bnip3 mediates GANT61-induced autophagy}

Although Bcl-2 family proteins were initially characterized as cell apoptosis regulators, it has recently become clear that they also control autophagy, playing a dual role in the regulation of apoptosis and autophagy. Thus, we assessed whether GANT61-induced autophagy might be associated with one or more Bcl-2 family proteins. As shown in Figure $4 \mathrm{~A}$, we observed robust upregulation of the BH3-only protein, Bnip3, by GANT61 in all three HCC cell lines; the expression of other Bcl-2 family proteins (including Bim, Noxa, Puma, Bcl2, and Bclxl) were not significantly affected, although the level of Mcl-1 was 
slightly reduced in two of the three HCC cell lines. GANT61 induced a 4.39-fold; 2.84-fold and 1.97-fold increase in Bnip3 mRNA level in Huh7, Hep3B and HepG2 cells, respectively, as determined by qRT-PCR (Figure 4B). The effect of GANT61 on Bnip3 expression was dose-dependent (at $24 \mathrm{~h}$ and $48 \mathrm{~h}$ time points) (Figure $4 \mathrm{C}$ ). The role of Bnip3 in GANT61-induced autophagy was supported by the observations that siRNA knockdown of Bnip3 prevented GANT61-induced LC3II accumulation (Figure 4D, left panel) and that overexpression of Bnip3 enhanced GANT61-induced LC3II accumulation (Figure 4D, right panel) and reversed SAG-induced LC3II reduction (Figure 4E).

We sought to further investigate the mechanism by which Hh signaling regulates Bnip3. As the Bnip3 promoter does not contain the Gli consensus DNA-binding sequences, it is likely that Hh signaling might regulate Bnip3 through an indirect mechanism. Given that Bnip3 is a downstream target of the MEK/ERK signaling pathway $(11,12)$ and that Hh and MEK/ ERK signaling pathways are known to interconnect in other cells(13-15), we performed experiments to determine whether inhibition of Hh by GANT61 might induce Bnip3 expression via activation of MEK/ERK. As shown in Figure 4F, GANT61 treatment increased the phosphorylation of MEK and ERK1/2 (but did not affect the levels of total MEK and ERK1/2). We observed that inhibition of MEK by U0126 prevented GANT61induced phosphorylation of ERK1/2, expression of Bnip3, and accumulation of LC3II (Figure 4G). These findings suggest that GANT61-induced Bnip3 expression is mediated at least in part through activation of the MEK/ERK pathway. Although Bnip expression is known to be regulated by NF- $\mathrm{kB}(16)$, p53(17), and DNA methyltransferase-1 (DNMT-1) (18), these molecules were not altered by GANT61 treatment in our system (Supplemental Figure S3).

\section{Bnip3 mediates GANT61-induced Beclin-1 dissociation from Bcl-2}

Beclin-1, the mammalian orthologue of yeast Atg6, is a well-known key regulator of autophagy; it is a critical component of the class III phosphatidylinositol-3-kinase complex (PI3KC3) required for autophagy. The overall structure of Beclin-1/Atg6 and its essential role in autophagosome formation is evolutionarily conserved throughout all eukaryotic phyla. Whereas Beclin-1 expression promotes autophagy, Beclin-1 reduction decreases autophagic activity(19). Sequence alignment and structural modeling indicate that Beclin-1 contains a putative BH3-like domain (a 112-123), which is known as a novel BH-3 domain only protein(20). The BH-3 domain of Beclin-1 interacts with Bcl-2, which leads to inhibition of autophagy $(21,22)$. Given that Bnip3 is a BH3- only protein and that GANT61 significantly up-regulated Bnip3 expression in HCC cells, we speculated that Bnip3 upregulation by GANT61 might cause Beclin-1 dissociation from Bcl-2. Indeed, as shown in Figure 5A and B, GANT61 treatment enhanced Bnip3 binding to Bcl-2 and caused Beclin-1 dissociation from Bcl-2. The role of Bnip3 in Beclin-1-Bcl-2 dissociation is further supported by the observations that forced overexpression of Bnip3 augmented GANT61induced Beclin-1 disassociation from Bcl-2 and that siRNA knockdown of Bnip3 partially reversed GANT61-induced Beclin-1 disassociation from Bcl-2 (Figure 5C). Consistent with these findings, forced overexpression of Bcl-2 was found to reduce GANT61-induced autophagy in Huh7 cells (Figure 5D). Taken together, these results indicate that the Gli inhibitor, GANT61, up-regulates Bnip3 expression and thus increases Bnip3 association with Bcl-2, which subsequently leads to Beclin-1 dissociation from Bcl-2 and induction of autophagy (illustrated in Figure 5E).

\section{GANT61-induced autophagy contributes to induction of apoptosis}

Autophagy is an evolutionarily conserved catabolic process that is thought to promote cell survival in response to stress. However, prolonged or excessive autophagy has also been shown to result in cell death under certain conditions (termed type II programmed cell 
death $)(10,23)$. To date, it remains unclear whether autophagy acts fundamentally as a cell survival or cell death pathway, or both. To investigate whether GANT61-induced autophagy might contribute to cell survival or death, we analyzed parameters of cell viability and apoptosis. We observed that GANT61 induced the cleavage of caspase-3, 8, 9 and PARP in Huh-7 cells, as determined by the Western blotting analysis (Figure 6A, left panel). Hoechst 33342 staining showed chromatin hypercondensation or fragmentation of nuclei in GANT61-treated Huh7 cells, which are characteristic features of apoptosis (Figure 6A, right panel). Consistent with these findings, GANT61 treatment decreased cell viability (as determined by WST1 assay) and reduced clonogenic survival capacity (Figure 6B). On the other hand, treatment with the Hh signaling agonists (SAG or Pur) enhanced cell growth and clonogenic survival capacity (Figure 6B). Treatment with the autophagic sequestration inhibitor, 3-MA, attenuated GANT61-induced apoptosis and reduction of cell viability and clonogenic survival capacity (Figure 6C). The pan-caspase inhibitor, zVAD-fmk, failed to block GANT61-induced autophagy (Figure 6D). These observations suggest that GANT61induced autophagy precede the execution of apoptosis.

Given the role of Bnip3 in GANT61-induced autophagy, we further examined the role of Bnip3 in GANT61-induced apoptosis. As shown in Figure 6E, knockdown of Bnip3 by siRNA prevented GANT61-induced apoptosis and cytotoxicity. Similarly, siRNA knockdown of Beclin-1 also prevented GANT61-induced apoptosis and cytotoxicity (Figure $6 \mathrm{~F})$. Therefore, GANT61-induced autophagy is not a protective mechanism against apoptosis in HCC cells; rather, it contributes to the induction of apoptosis.

\section{Inhibition of autophagy by 3-MA and CQ prevents GANT61-induced apoptosis}

Apoptosis and autophagy are tightly regulated biological processes and their cross-talk is complex, with conflicting models of interplays (partnership or antagonist) being indicated(10). To gain insight into variable status between autophagy and apoptosis, we compared the apoptotic effect of GANT61 with other chemotherapeutic agents which have been reported to have cytotoxic effects in HCC cells at indicated concentrations (listed in Supplemental Table 1). As shown in Figure 7A, inhibition of autophagy by 3-MA and CQ partially reversed the cytotoxic effect induced by GANT61, sorafenib (a FDA approved multikinase inhibitor for treatment of HCC in patients) and other chemotherapeutic/ chemopreventive agents in Huh7 cells. However, in HepG2 and Hep3B cells, 3-MA and CQ exhibited variable effects depending on specific chemotherapeutic/chemopreventive agents. Thus, autophagy may contribute to cell survival or death depending on specific context and different cell types. We observed that that 3-MA and CQ prevented cytotoxicity induced by GANT61 and sorafenib consistently in all three cell lines. The latter finding is consistent with the data of flow cytometry using Annexin-V/propidium iodide staining showing that inhibition of autophagy by 3-MA and CQ prevented GANT61 and sorafenib-induced apoptosis in Huh7 cells (Figure 7B). These results are noteworthy, given the role of the Gli inhibitor GANT61 for induction of autophagy and apoptosis in HCC cells as documented in the current study and the fact that sorafenib is the only therapeutic agent currently available for systemic therapy of HCC in patients.

\section{GANT61 induces autophagy and suppresses HCC growth in vivo}

To assess the antitumor potential of GANT61 and the role of autophagy in vivo, we employed a tumor xenograft model in which Huh7 cells were inoculated into SCID mice and the animals were treated intraperitoneally with vehicle control, GANT61, or GANT61 in combination with 3-MA (i.p. injection, started one week after inoculation, performed every other day for 4 weeks). As shown in Figure 8A and 8B, GANT61 treatment significantly inhibited Huh7 tumor growth and the effect was attenuated by the autophagy inhibitor, 3-MA. Induction of autophagy in GANT61-treated tumor was confirmed by 
immunofluorescent staining and immunoblotting for LC3II (Figure 8C and 8D). GANT61 treatment increased the cleavage of caspase- 3 and caspase- 8 in tumor tissues and the effect was partially reversed by co-treatment with 3-MA. These results suggest that GANT61induced autophagy contribute to HCC cell apoptosis and cytotoxicity in vivo and that the activity of autophagy is a key factor that determines the efficacy of Hh-targeted therapy.

\section{DISCUSSION}

This study provides novel evidence that Hh signaling pathway is a key regulator of autophagy in HCC cells. Although Hh signaling activation in HCC cells may exert effect on other cell types in the liver (such as hepatic stellate cells, liver progenitor cells, and tumorinitiating stem-like cells)(24), our data provide the first evidence for an autocrine action of Hh signaling in HCC cells. Our data show that inhibition of Gli by GANT61 induces autophagy, whereas activation of $\mathrm{Hh}$ signaling by its ligand and agonists inhibits autophagic activity. This process is mediated by Bnip3, which displaces Bcl-2 from Beclin-1. Moreover, our data show that inhibition of autophagy attenuates GANT61-induced apoptosis. These findings provide the first evidence that $\mathrm{Hh}$ signaling regulates autophagy and that autophagic activity is a key factor that determines cell response to Hh-targeted therapy.

We have found that GANT61-induced autophagy is mediated through upregulation of Bnip3, which displaces Bcl-2 from Beclin-1. The Bcl-2 family of proteins is an important regulator of both apoptosis and autophagy and contains both anti- and pro-apoptotic members(20). The anti-apoptotic members (e.g., Bcl-2, Bcl-xL and Mcl-1) protect cells from apoptosis and contain characteristic regions of Bcl-2 homology (BH) domains (BH1, $\mathrm{BH} 2, \mathrm{BH} 3$ and $\mathrm{BH} 4)$. The pro-apoptotic members of the family are divided into two subgroups: proteins that contain two or three $\mathrm{BH}$ domains; and proteins that contain only $\mathrm{BH}$, the domain essential for binding to the anti-apoptotic members of the family (so-called BH3-only proteins). The BH3-only proteins (such as Noxa, Bad, Bnip3, and Puma) act as sentinels of stress or damage and are key instigators of cell death in many situations(25); they are also known to induce autophagy(10). Beclin-1, a key player in the initiation of autophagy, was recently identified as a new member of the BH3-only proteins (the BH3 domain of Beclin-1 interacts with Bcl-2 and this interaction leads to suppression of autophagy) $(21,22)$. In this study, we found that inhibition of Gli by GANT61 significantly increased the protein and mRNA levels of Bnip3 in all three HCC cell lines and that Bnip3 induced dissociation of the Beclin-1/Bcl-2 binding complex. Our findings suggest a model in which inhibition of Hh signaling causes upregulation of Bnip3 and this leads to dissociation of the Beclin-1/Bcl-2 binding complex and subsequent induction of autophagy. In spite of the robust upregulation of Bnip3 by GANT61 in all three HCC cell lines, the expression of other Bcl-2 family proteins was not significantly affected, except for Mcl-1. In our system, the level of Mcl-1 was slightly reduced by GANT61 treatment in two of the three HCC cell lines. It remains to be determined whether Mcl-1 reduction might also contribute to GANT-induced HCC cell apoptosis, although it is beyond the scope of the current study. Further investigations are warranted to dissect the emerging connections between Hh signaling and the Bcl-2 family proteins.

Several molecules have been implicated in the modulation of Bnip3 expression, including MEK/ERK(11, 12), NF- $\mathrm{BB}(16), \mathrm{p53}(17)$, and methylation of Bnip3 promoter by DNAmethyltransferase 1(18). In the current study, we observed that GANT61 treatment activated the MEK/ERK signaling, as reflected by increased phospho-MEK and phospho-ERK1/2. The MEK inhibitor, U0126, prevented GANT61-induced upregulation of Bnip3 expression and LC3 accumulation. These observations suggest that Hh inhibition by GANT61 upregulates the expression of Bnip3, at least in part, through activation of the MEK/ERK 
signaling pathway. As activation or inhibition of Hh signaling pathway did not affect the levels of NF- $\mathrm{kB}$, p53 and DNMT1/DNMT3a, these molecules do not appear to be involved in GANT61-induced upregulation of Bnip3 in HCC cells.

The role of autophagy in caner development and progression is complex. Whereas deficiency of autophagy can predispose to the initiation of tumor development, excessive or prolonged activation of autophagy may promote cancer cell death(10). Paradoxically, autophagy is also known to enhance cancer cell survival in response to some environmental and cellular stresses (e.g., nutrient deprivation, organelle damage, hypoxia, or therapeutic stress) and causes resistance to antineoplastic therapies. In this study, we attempted to explore whether autophagy induced by GANT61 in HCC cells is a cell death or survival mechanism. We provided in vitro and in vivo evidences that inhibition of Gli by GANT61 induces both autophagy and apoptosis in HCC cells and that blockage of autophagy reverses GANT61-induced apoptosis and cytotoxicity. Although the role of autophagy in cell survival and death may depend on specific agents and cell types, our data clearly demonstrate that autophagy contributes to HCC cell apoptosis induced by the Gli inhibitor GANT61, a promising new anti-cancer drug, and by the mulikinase inhibitor sorafenib, the only FDA-approved drug for target therapy of HCC.

Inhibition of Hh signaling has been attempted in various human cancer models. Several natural and synthetic pharmacologic agents for modulation of Hh activity have been identified and developed. Historically, Smo antagonists including cyclopamine and GDC-0449, respectively, have been used to abrogate Hh signaling in human cancers with moderate success. A potentially more potent target lies in the family of Gli transcription factors, which are the final arbiters of transcriptional regulation in the Hh signaling pathway(26). GANT61 is a recently identified small molecule inhibitor of Gli, which has been shown to effectively block Gli-1 and Gli-2 activities and induce more significant cytotoxicity in human cancer cells than Smo antagonists(26). In HCC cells, we observed that the Gli inhibitor, GANT61, induced more prominent autophagy and apoptotic cell death compared to the Smo inhibitor, GDC-0449.

In conclusion, this study shows that Hh signaling pathway importantly regulates autophagy and that inhibition of $\mathrm{Hh}$ signaling activates autophagy in human hepatocellular carcinoma cells at least in part through induction of Bnip3 which prevents Beclin-1 binding to Bcl-2. Furthermore, we show that autophagy contributes to GANT61-induced apoptosis and inhibition of growth in $\mathrm{HCC}$ cells. These findings provide the first evidence that $\mathrm{Hh}$ signaling regulates autophagy and that autophagic activity is a key factor that determines cell response to Hh-targeted therapy.

\title{
Supplementary Material
}

Refer to Web version on PubMed Central for supplementary material.

\section{Acknowledgments}

The authors thank the Louisiana Cancer Research Consortium FACS Core facility for flow cytometry analysis. Supported by National Institutes of Health grants CA106280, CA102325, CA134568, and DK077776 (to T.W.).

\author{
Abbreviations \\ HCC hepatocellular carcinoma \\ Hh hedgehog
}




$\begin{array}{ll}\text { Smo } & \text { smoothened } \\ \text { LC3 } & \text { microtubule-associated protein light chain 3 } \\ \text { Pur } & \text { purmorphamine } \\ \text { ATG } & \text { autophagy-related gene } \\ \text { BH domain } & \text { Bcl-2 homology domain } \\ \text { MDC } & \text { monodansylcadaverine } \\ \text { TEM } & \text { transmission electron microscopy } \\ \text { 3-MA } & \text { 3-methyladenine } \\ \text { CBZ } & \text { carbamazepine } \\ \text { Ptc } & \text { patched } \\ \text { CQ } & \text { chloroquine } \\ \text { GFP } & \text { green fluorescence protein } \\ \text { SCID } & \text { severe combined immune-deficiency }\end{array}$

\section{REFERENCES}

1. Nusslein-Volhard C, Wieschaus E. Mutations affecting segment number and polarity in Drosophila. Nature. 1980; 287:795-801. [PubMed: 6776413]

2. Jiang J, Hui CC. Hedgehog signaling in development and cancer. Dev Cell. 2008; 15:801-812. [PubMed: 19081070]

3. Ingham PW, McMahon AP. Hedgehog signaling in animal development: paradigms and principles. Genes Dev. 2001; 15:3059-3087. [PubMed: 11731473]

4. Huang S, He J, Zhang X, Bian Y, Yang L, Xie G, Zhang K, et al. Activation of the hedgehog pathway in human hepatocellular carcinomas. Carcinogenesis. 2006; 27:1334-1340. [PubMed: 16501253]

5. Sicklick JK, Li YX, Jayaraman A, Kannangai R, Qi Y, Vivekanandan P, Ludlow JW, et al. Dysregulation of the Hedgehog pathway in human hepatocarcinogenesis. Carcinogenesis. 2006; 27:748-757. [PubMed: 16339184]

6. Yang JD, Roberts LR. Hepatocellular carcinoma: A global view. Nat Rev Gastroenterol Hepatol. 2010; 7:448-458. [PubMed: 20628345]

7. Rubin LL, de Sauvage FJ. Targeting the Hedgehog pathway in cancer. Nat Rev Drug Discov. 2006; 5:1026-1033. [PubMed: 17139287]

8. Mizushima N. Autophagy: process and function. Genes Dev. 2007; 21:2861-2873. [PubMed: 18006683]

9. Maiuri MC, Zalckvar E, Kimchi A, Kroemer G. Self-eating and self-killing: crosstalk between autophagy and apoptosis. Nat Rev Mol Cell Biol. 2007; 8:741-752. [PubMed: 17717517]

10. Elgendy M, Sheridan C, Brumatti G, Martin SJ. Oncogenic Ras-induced expression of Noxa and Beclin-1 promotes autophagic cell death and limits clonogenic survival. Mol Cell. 2011; 42:23-35. [PubMed: 21353614]

11. An HJ, Maeng O, Kang KH, Lee JO, Kim YS, Paik SG, Lee H. Activation of Ras up-regulates proapoptotic BNIP3 in nitric oxide-induced cell death. J Biol Chem. 2006; 281:33939-33948. [PubMed: 16954213]

12. Wu SY, Lan SH, Cheng DE, Chen WK, Shen CH, Lee YR, Zuchini R, et al. Ras-related tumorigenesis is suppressed by BNIP3-mediated autophagy through inhibition of cell proliferation. Neoplasia. 2011; 13:1171-1182. [PubMed: 22241963] 
13. Mimeault M, Batra SK. Frequent deregulations in the hedgehog signaling network and cross-talks with the epidermal growth factor receptor pathway involved in cancer progression and targeted therapies. Pharmacol Rev. 2010; 62:497-524. [PubMed: 20716670]

14. Schnidar H, Eberl M, Klingler S, Mangelberger D, Kasper M, Hauser-Kronberger C, Regl G, et al. Epidermal growth factor receptor signaling synergizes with Hedgehog/GLI in oncogenic transformation via activation of the MEK/ERK/JUN pathway. Cancer Res. 2009; 69:1284-1292. [PubMed: 19190345]

15. Rajurkar M, De Jesus-Monge WE, Driscoll DR, Appleman VA, Huang H, Cotton JL, Klimstra DS, et al. The activity of Gli transcription factors is essential for Kras-induced pancreatic tumorigenesis. Proc Natl Acad Sci U S A. 2012; 109:E1038-E1047. [PubMed: 22493246]

16. Baetz D, Regula KM, Ens K, Shaw J, Kothari S, Yurkova N, Kirshenbaum LA. Nuclear factorkappaB-mediated cell survival involves transcriptional silencing of the mitochondrial death gene BNIP3 in ventricular myocytes. Circulation. 2005; 112:3777-3785. [PubMed: 16344406]

17. Feng X, Liu X, Zhang W, Xiao W. p53 directly suppresses BNIP3 expression to protect against hypoxia-induced cell death. EMBO J. 2011; 30:3397-3415. [PubMed: 21792176]

18. An HJ, Lee H, Paik SG. Silencing of BNIP3 results from promoter methylation by DNA methyltransferase 1 induced by the mitogen-activated protein kinase pathway. Mol Cells. 2011; 31:579-583. [PubMed: 21573703]

19. Liang XH, Jackson S, Seaman M, Brown K, Kempkes B, Hibshoosh H, Levine B. Induction of autophagy and inhibition of tumorigenesis by beclin 1. Nature. 1999; 402:672-676. [PubMed: 10604474]

20. Levine B, Sinha S, Kroemer G. Bcl-2 family members: dual regulators of apoptosis and autophagy. Autophagy. 2008; 4:600-606. [PubMed: 18497563]

21. Pattingre S, Tassa A, Qu X, Garuti R, Liang XH, Mizushima N, Packer M, et al. Bcl-2 antiapoptotic proteins inhibit Beclin 1-dependent autophagy. Cell. 2005; 122:927-939. [PubMed: 16179260]

22. Maiuri MC, Le Toumelin G, Criollo A, Rain JC, Gautier F, Juin P, Tasdemir E, et al. Functional and physical interaction between Bcl-X(L) and a BH3-like domain in Beclin-1. EMBO J. 2007; 26:2527-2539. [PubMed: 17446862]

23. Kroemer G, Levine B. Autophagic cell death: the story of a misnomer. Nat Rev Mol Cell Biol. 2008; 9:1004-1010. [PubMed: 18971948]

24. Omenetti A, Choi S, Michelotti G, Diehl AM. Hedgehog signaling in the liver. J Hepatol. 2011; 54:366-373. [PubMed: 21093090]

25. Strasser A. The role of BH3-only proteins in the immune system. Nat Rev Immunol. 2005; 5:189200. [PubMed: 15719025]

26. Lauth M, Bergstrom A, Shimokawa T, Toftgard R. Inhibition of GLI-mediated transcription and tumor cell growth by small-molecule antagonists. Proc Natl Acad Sci U S A. 2007; 104:84558460. [PubMed: 17494766] 
A

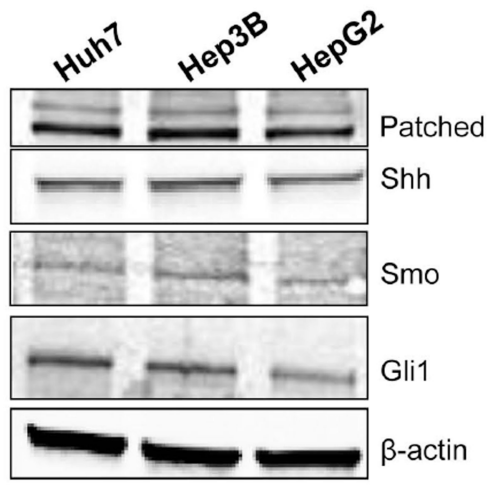

C

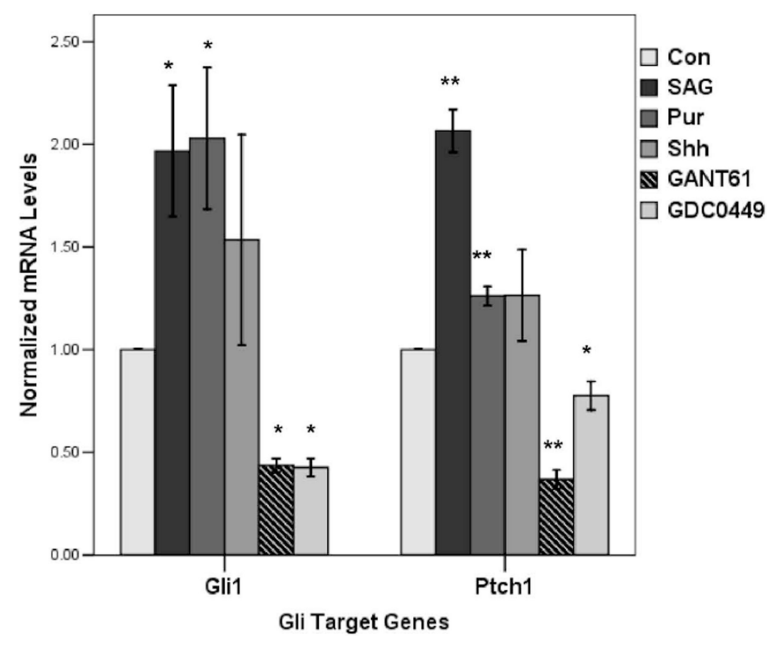

B

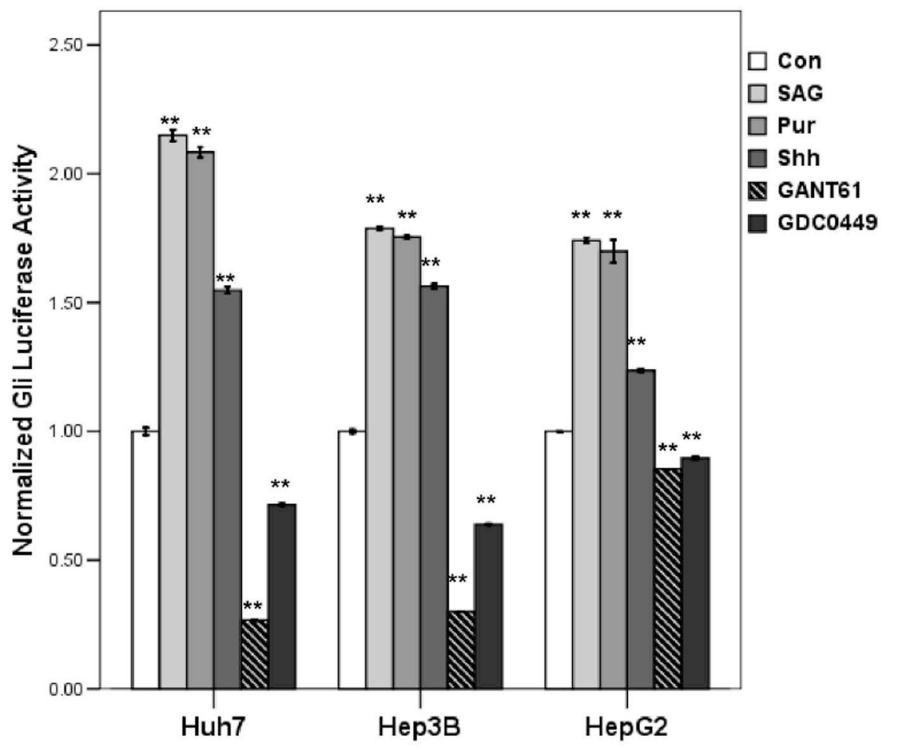

Figure 1. Hh signaling pathway is active in human hepatocellular carcinoma cells

(A) Western blotting for Shh, Patched, Smo, and Gli1 in Huh7, Hep3B, HepG2 cells. (B) Gliluciferase reporter activities. The cells were transfected Gli-luciferase reporter plasmid with Renilla at 20:1 ratio, and then treated for 24 hours with vehicle control, SAG $(0.5 \mu \mathrm{M})$, Pur $(10 \mu \mathrm{M})$, Shh $(0.4 \mu \mathrm{g} / \mathrm{ml})$, GANT61 $(20 \mu \mathrm{M})$ or GDC0449 $(20 \mu \mathrm{M})$, respectively $(\mathrm{n}=3$; $* * \mathrm{p}<0.01$ compared to vehicle control). (C) mRNA levels of Ptch1 and Gli1 as determined by qRT-PCR. Huh7 cells were treated for 48 hours with vehicle control, SAG $(0.5 \mu \mathrm{M})$, Pur $(10 \mu \mathrm{M})$, Shh $(0.4 \mu \mathrm{g} / \mathrm{ml})$, GANT61 $(20 \mu \mathrm{M})$ or GDC0449 $(20 \mu \mathrm{M})$, respectively (the data were normalized to the level of actin mRNA; $n=3$; **p $<0.01, * \mathrm{p}<0.05$ compared to vehicle control). 
A
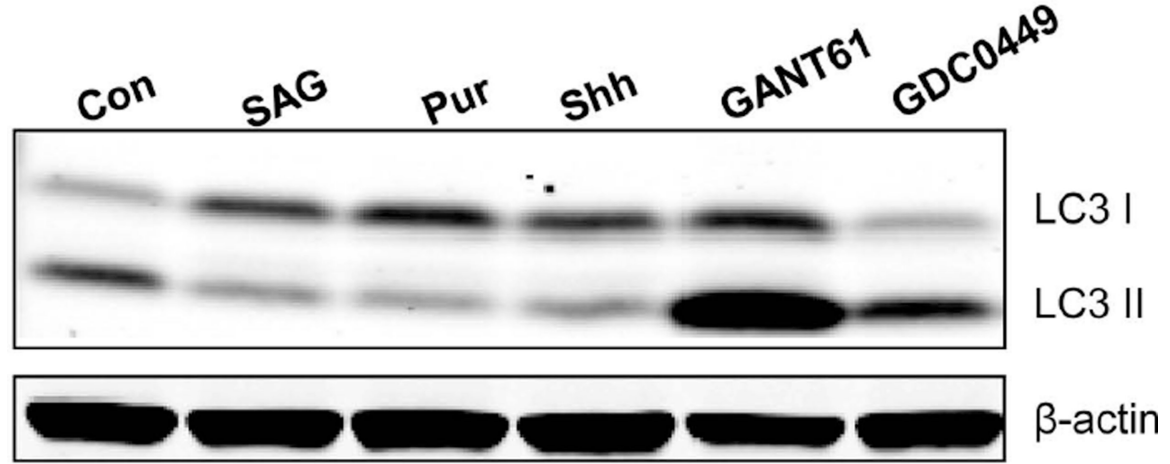

\section{Huh7}

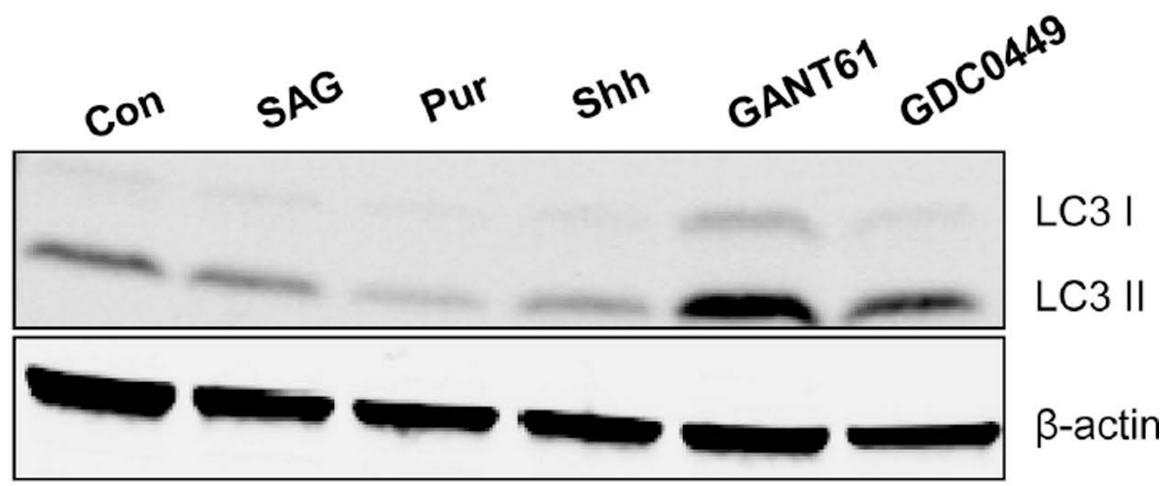

Hep3B

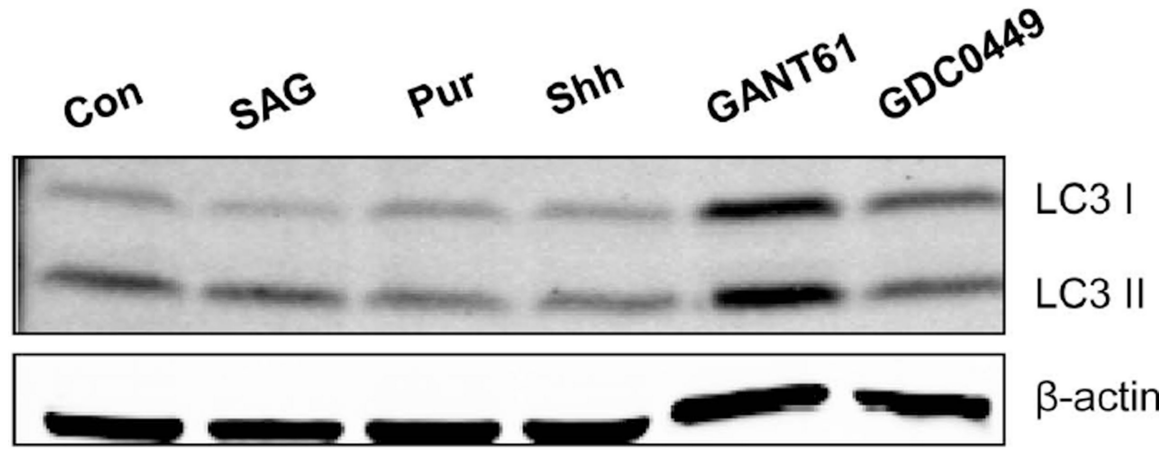

HepG2 


\section{B}

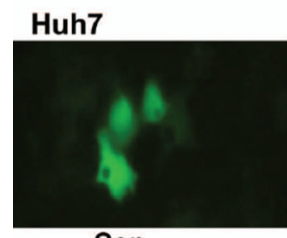

Con

Hep3B

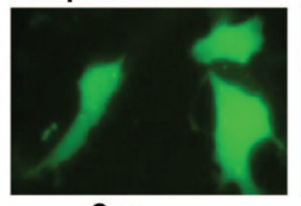

Con

HepG2

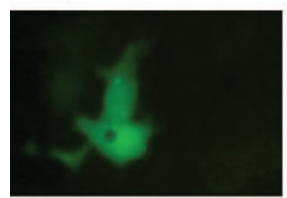

Con

C

GANT61 24h treatment

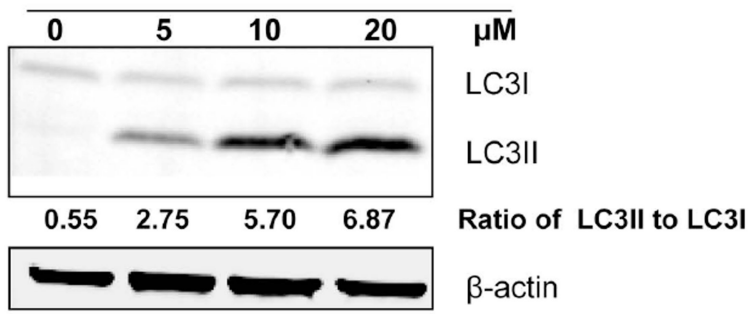

GANT61 48h treatment

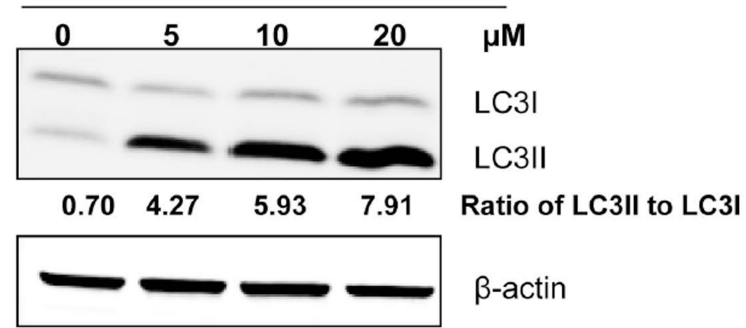

Pur

Pur

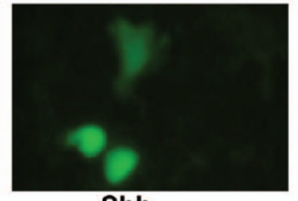

Shh
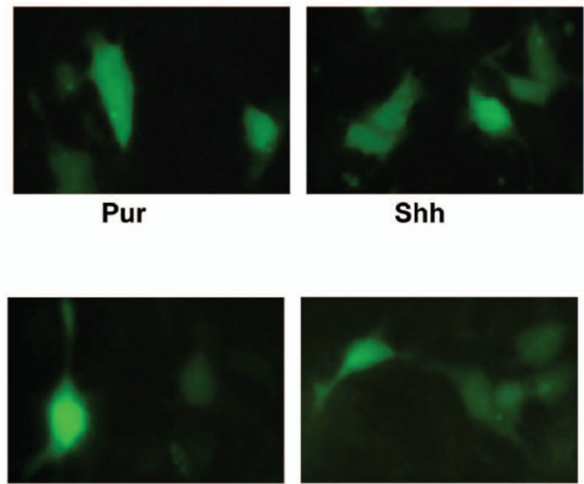

Shh

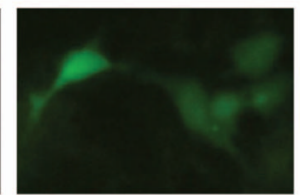

Shh

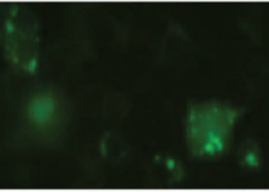

GANT61

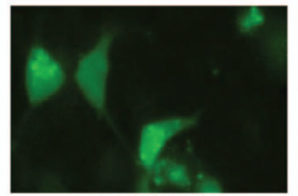

GANT61

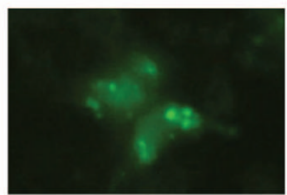

GANT61

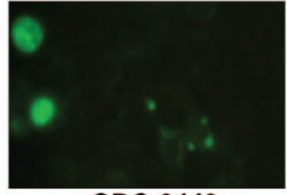

GDC-0449

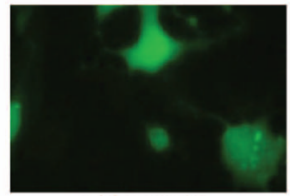

GDC-0449

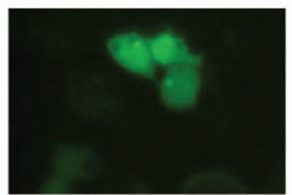

GDC-0449

D
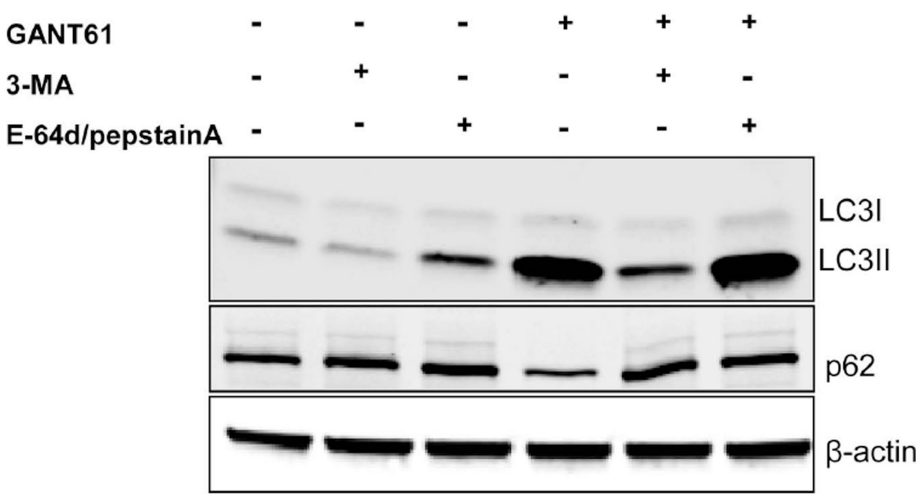

Figure 2. Inhibition of Hh signaling induces autophagy

(A) Western blotting for LC3I/II. Huh7, Hep3B and HepG2 cells were incubated with vehicle control, SAG $(0.5 \mu \mathrm{M})$, Pur $(10 \mu \mathrm{M})$, Shh $(0.4 \mu \mathrm{g} / \mathrm{ml})$, GANT61 (20 $\mu \mathrm{M})$ or GDC0449 $(20 \mu \mathrm{M})$ for 48 hours. (B) GFP-LC3 puncta as visualized by fluorescence microscopy. Huh7, Hep3B and HepG2 cells transfected with GFP-LC3 expressing plasmid were treated with SAG $(0.5 \mu \mathrm{M})$, Pur $(10 \mu \mathrm{M})$, Shh $(0.4 \mu \mathrm{g} / \mathrm{ml})$, GANT61 $(20 \mu \mathrm{M})$ or GDC0449 $(20 \mu \mathrm{M})$ for 24 hours. GFP-LC3-positive (>3 punctate staining sites per cell) cells 
was defined as an indicator of autophagy. (C) Western blotting for LC3I and II in Huh7 cells treated with GANT61. (D) Western blotting for LC3I/II and P62 in Huh7 cells treated with GANT61 for $24 \mathrm{~h}$ in the presence or absence of 3-MA ( $5 \mathrm{mmol} / \mathrm{L})$ or E-64d/pepstain A (10 $\mu \mathrm{g} / \mathrm{ml})$. 

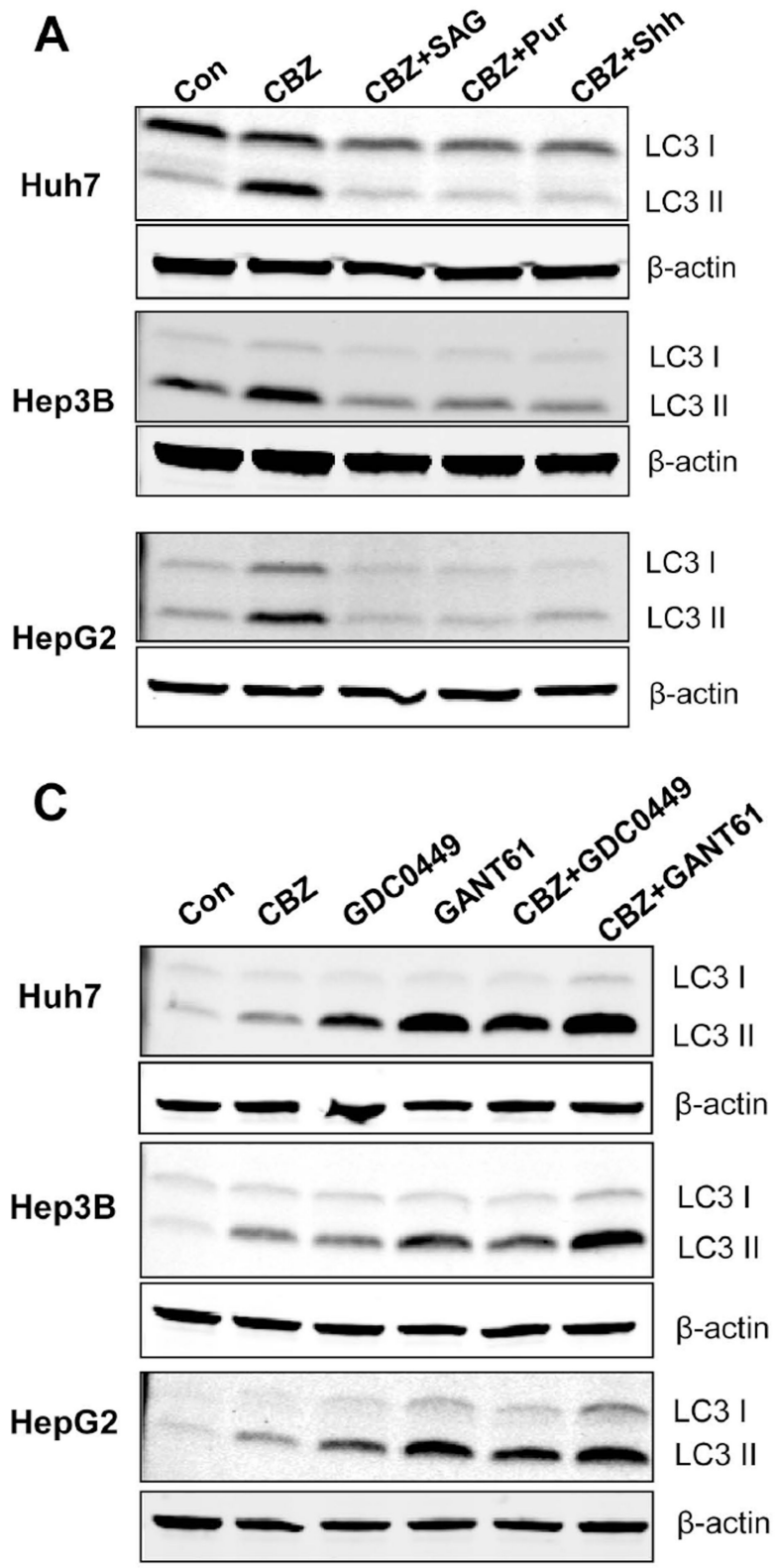
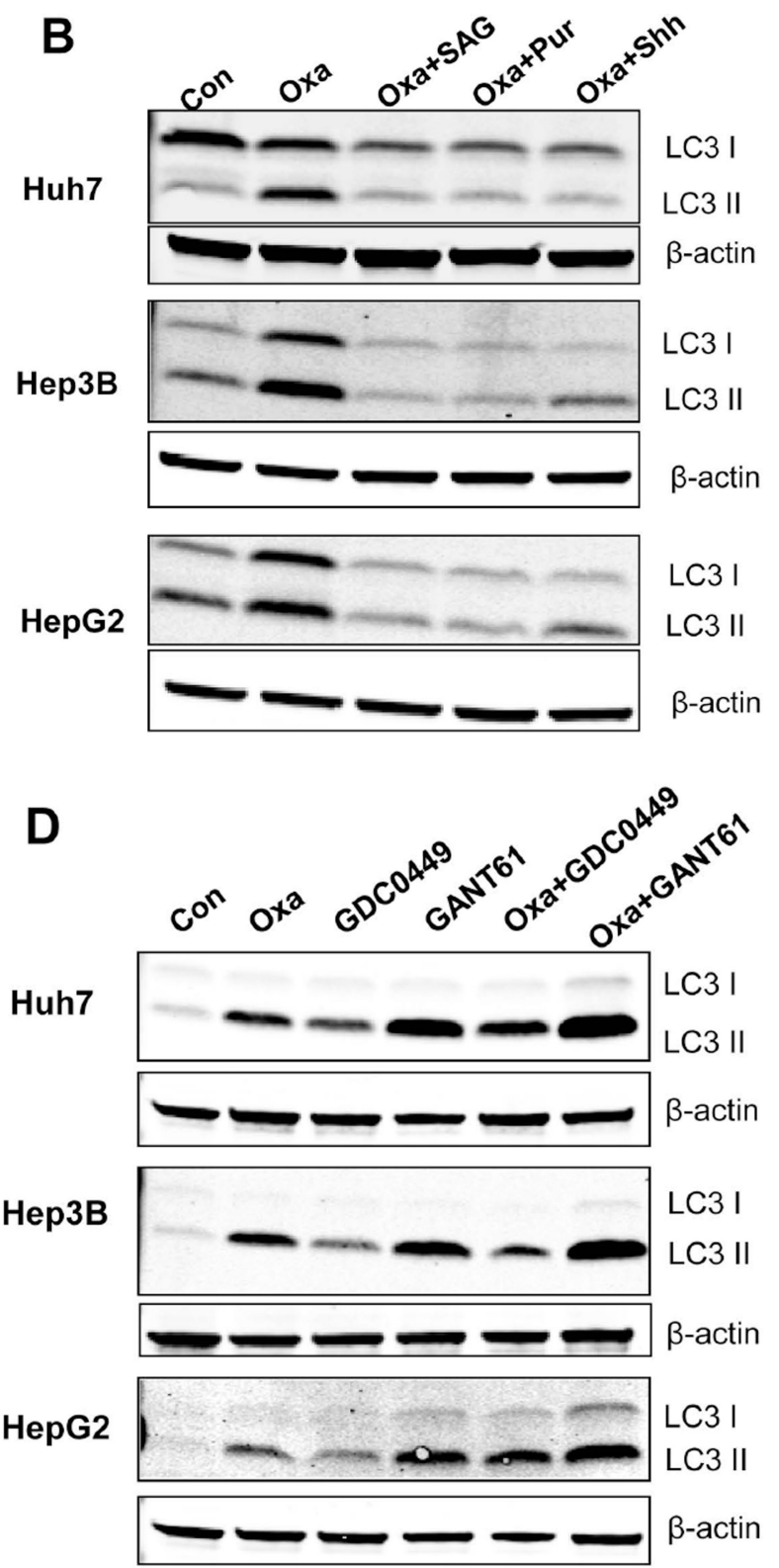

Figure 3. The effect of Hh signaling on drug-induced autophagy

Huh7, HepG2 and Hep3B cells grown in 6-wells plates were treated with $200 \mu \mathrm{M}$ carbamazepine (A, C) or $20 \mu \mathrm{M}$ oxaliplatin $(\mathbf{B}, \mathbf{D})$, respectively, in the absence or presence of the Hh signaling agonists $(0.5 \mu \mathrm{M} \mathrm{SAG}, 10 \mu \mathrm{M}$ Pur or $0.4 \mu \mathrm{g} / \mathrm{ml}$ Shh) or inhibitors (20 $\mu \mathrm{M}$ GDC0449 or GANT61). After $48 \mathrm{~h}$ incubation, the cell lysates were obtained for western blotting to detect LC3I/II. 
A
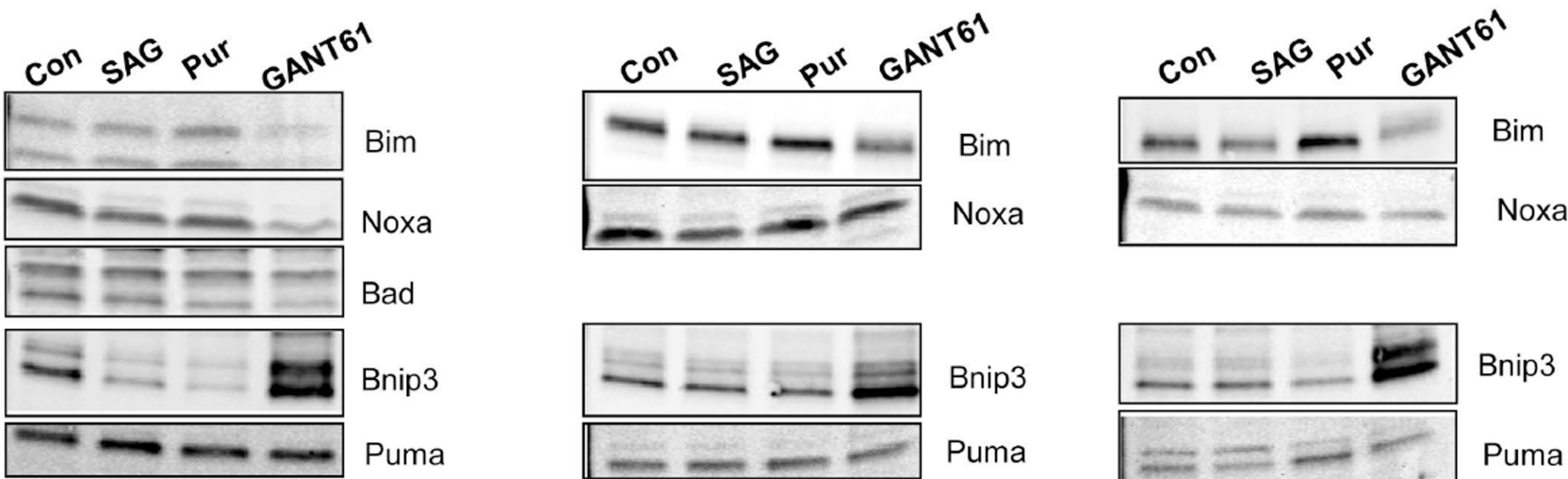

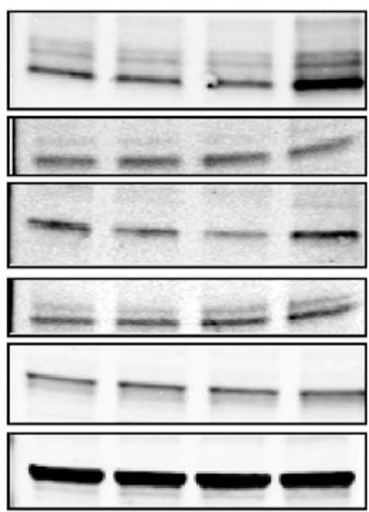

Hep3B

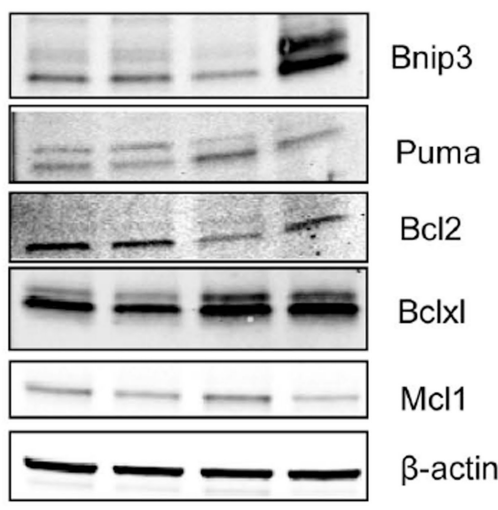

HepG2

B

C
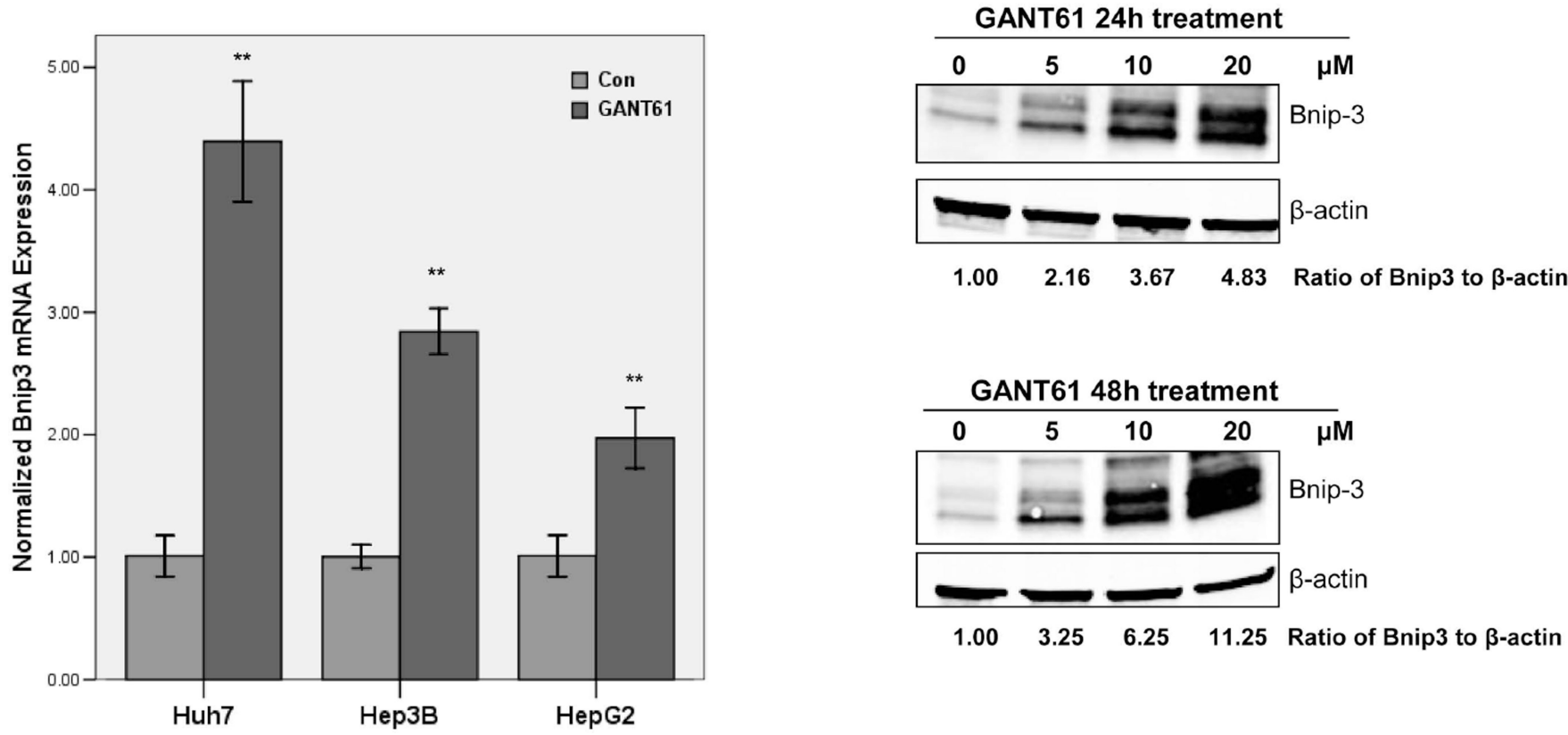
D

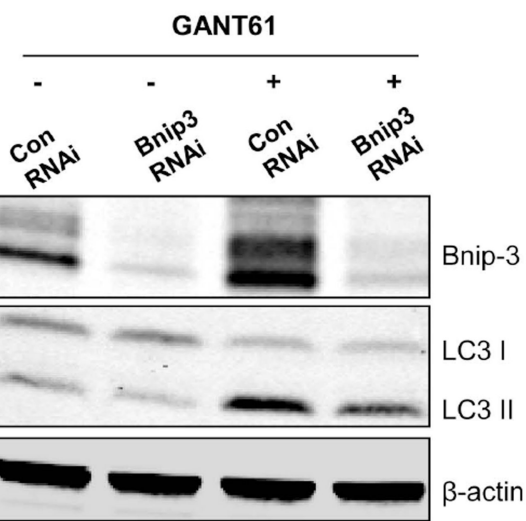

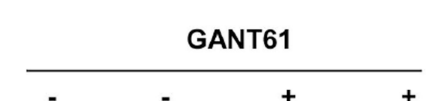

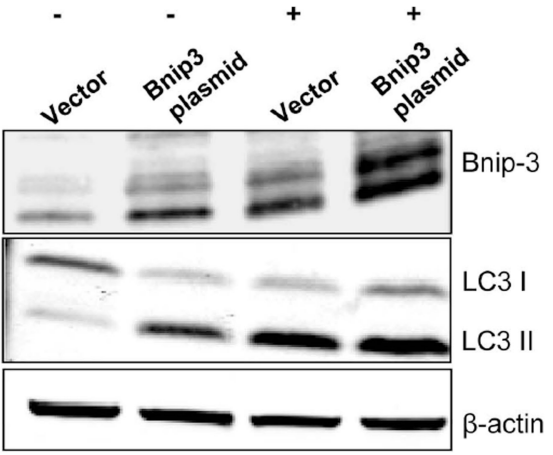

E
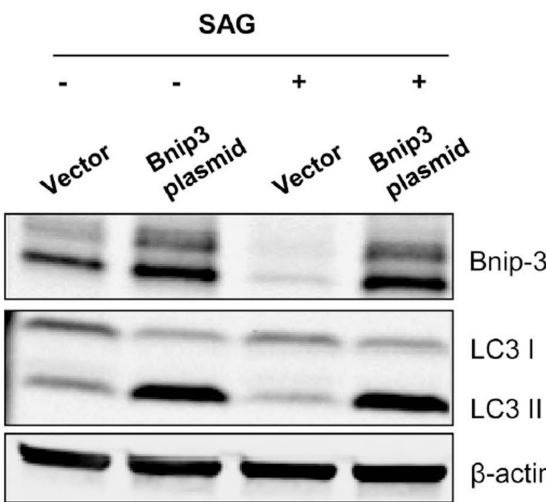

LC3 I

LC3 II

$\beta$-actin
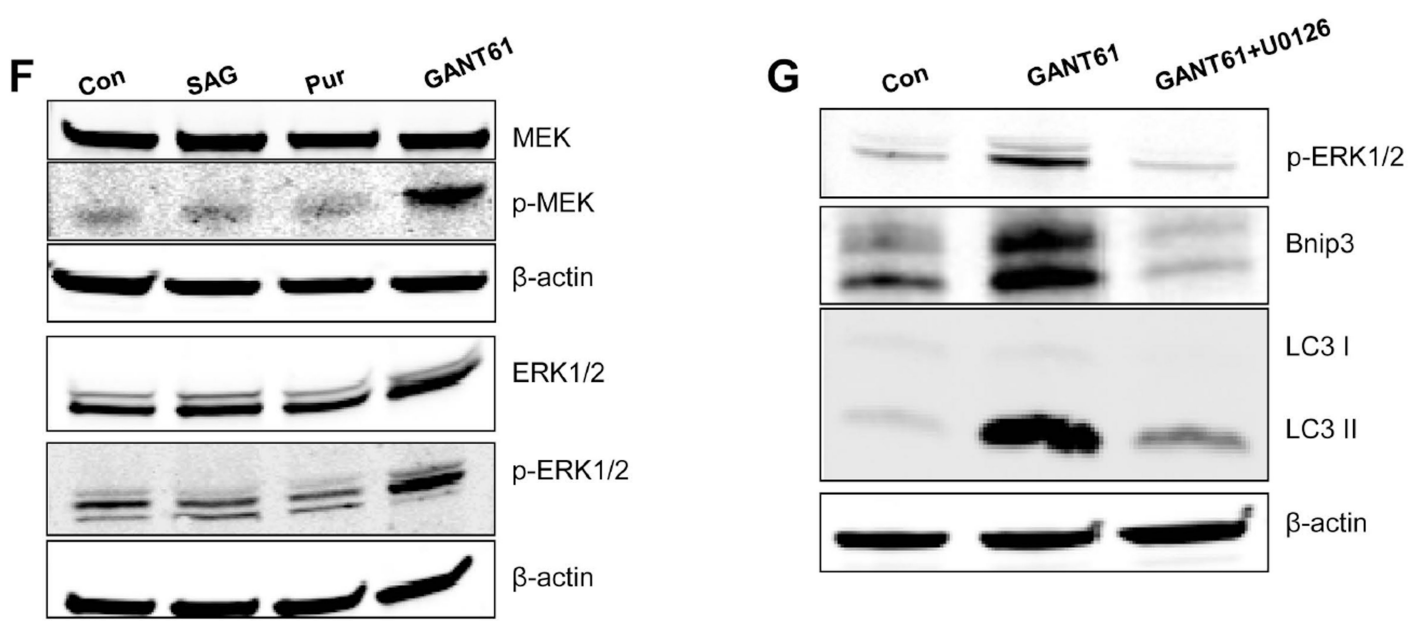

Figure 4. Bnip3 mediates GANT61-induced autophagy

(A) Western blotting for indicated Bcl-2 family proteins in Huh7, Hep3B and HepG2 cells treated with $0.5 \mu \mathrm{M}$ SAG, $10 \mu \mathrm{M}$ Pur or $20 \mu \mathrm{M}$ GANT61, respectively, for 48 hours (B) qRT-PCR for Bnip-3 mRNA in HCC cells treated with vehicle control or $20 \mu \mathrm{M}$ GANT61 for 48 hours (the data were normalized to actin mRNA; $n=3 ; * * p<0.01$ ). (C) Western blotting for Bnip3 in Huh7 cells treated with 0-20 $\mu$ M GANT61 for 24 and 48 hours. (D) Western blotting analysis for Bnip3 and LC3I/II. Huh7 cells were transfected with Bnip3 siRNA (left panel) or expression plasmid (right panel) for $6 \mathrm{~h}$ and the cells were treated with $20 \mu \mathrm{M}$ GANT61 for 48h. (E) Western blotting for Bnip3 and LC3I/II in Bnip3 overexpressed or control Huh7 cells treated with $0.5 \mu \mathrm{M}$ SAG for 48h. (F) Western blotting for MEK, ERK1/2, phosphor-MEK and phosphor-ERK1/2 in Huh7 cells treated with $0.5 \mu \mathrm{M}$ SAG, $10 \mu \mathrm{M}$ Pur or $20 \mu \mathrm{M}$ GANT61 for 48 hours. (G) Western blotting for phosphorERK1/2, Bnip3 and LC3 in Huh7 cells treated with $20 \mu \mathrm{M}$ GANT61 or $20 \mu \mathrm{M}$ GANT61 plus $10 \mu \mathrm{M}$ U0126. 
A
IP: Bcl2

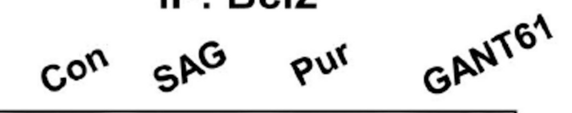

IB:Bnip3

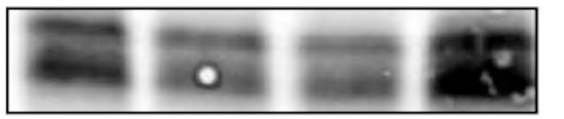

IP: IgG

IB: Bnip3

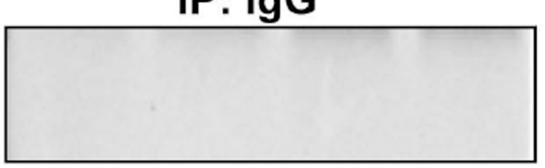

Input

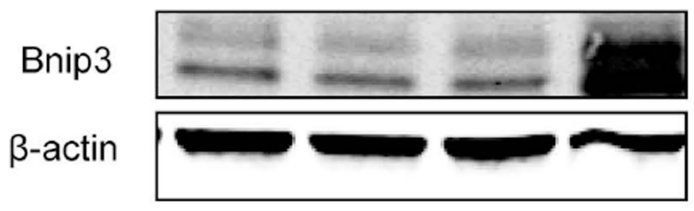

B

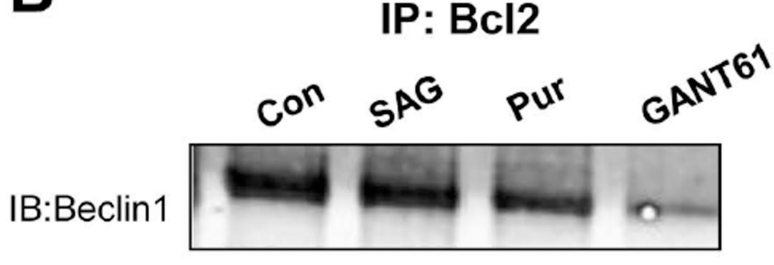

IP: IgG

IB:Beclin1

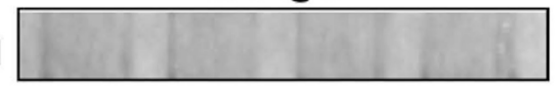

Input

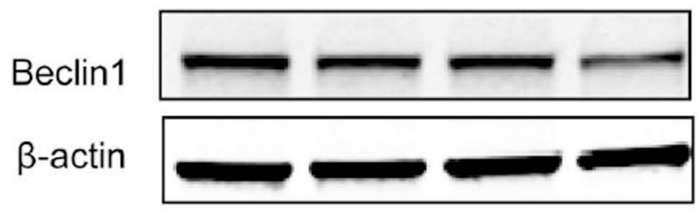

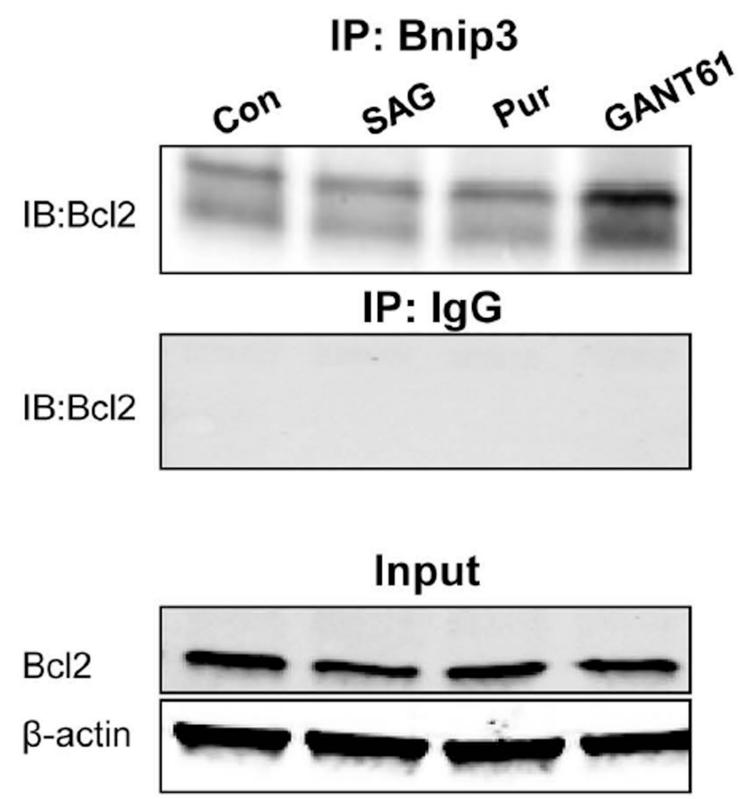

IP: Beclin1

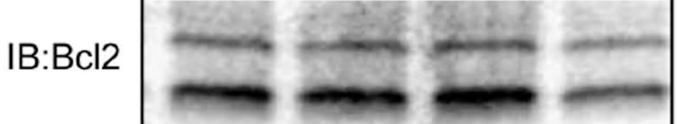

IP: IgG

$\mathrm{IB}: \mathrm{Bc} \mid 2$

Input

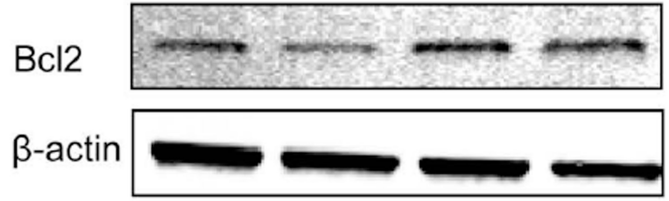


C

IP: Bcl2

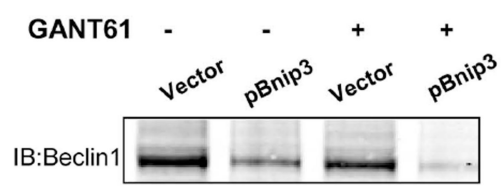

IP: $\lg G$
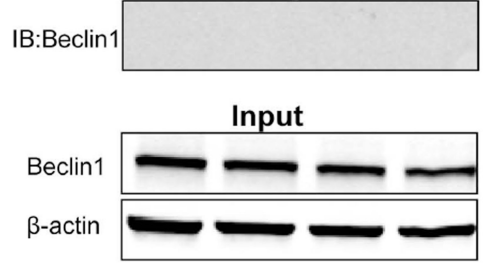

IP: Bcl2

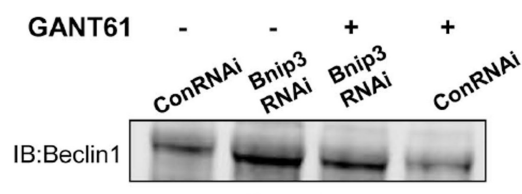

IP: $\lg G$

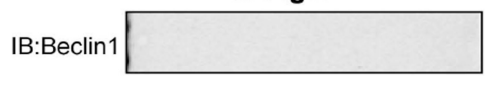

Input

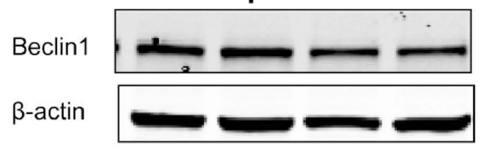

IP: Beclin1

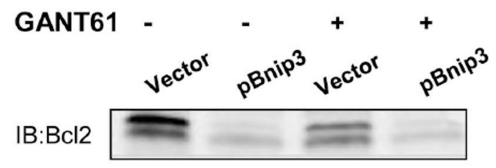

IP: IgG
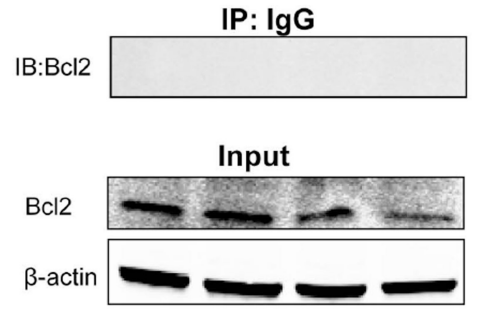

IP: Beclin1

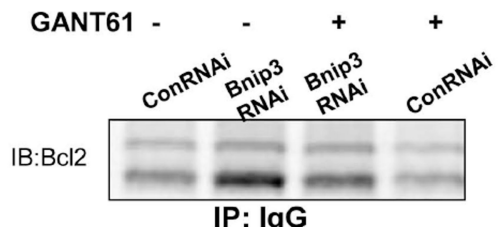

$\mathrm{IB}: \mathrm{Bcl} 2$

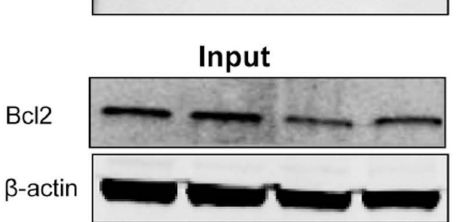

D

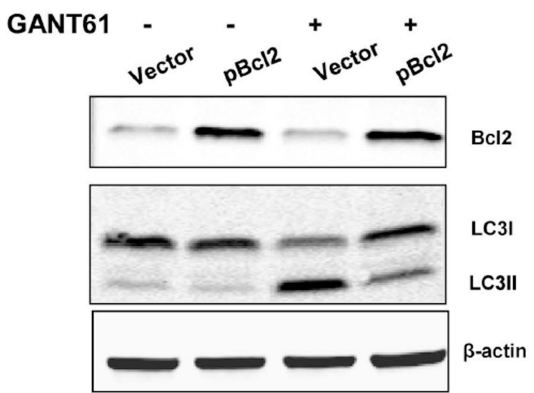

E

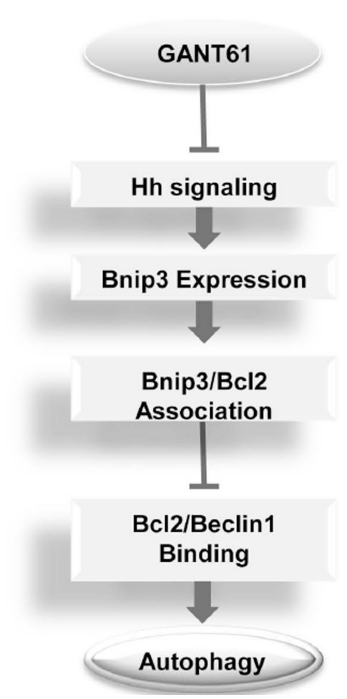

Figure 5. Bnip-3 mediates GANT61-induced Beclin-1 dissociation from Bcl-2

(A) Huh7 cells were treated with vehicle control, SAG, Pur or GANT61 for 48 hours and the cell lysates were obtained for immunoprecipitation and immunoblotting using indicated antibodies. (B) Huh7 cell were treated with indicated reagents for 48 hours and the cell lysates were obtained for immunoprecipitation and immunoblotting with indicated antibodies. (C) Huh7 cells were transfected with Bnip3 expressed plasmid (upper panel) or siRNA (lower panel) and the cell lysates were obtained for immunoprecipitation and immunoblotting using indicated antibodies. (D) Western blotting for LC3I/II in Huh7 cells transfected with Bcl-2 expressed plasmid or control vector. (E) Schematic diagram illustrating the mechanism of GANT61-induced autophagy. 


\section{A}
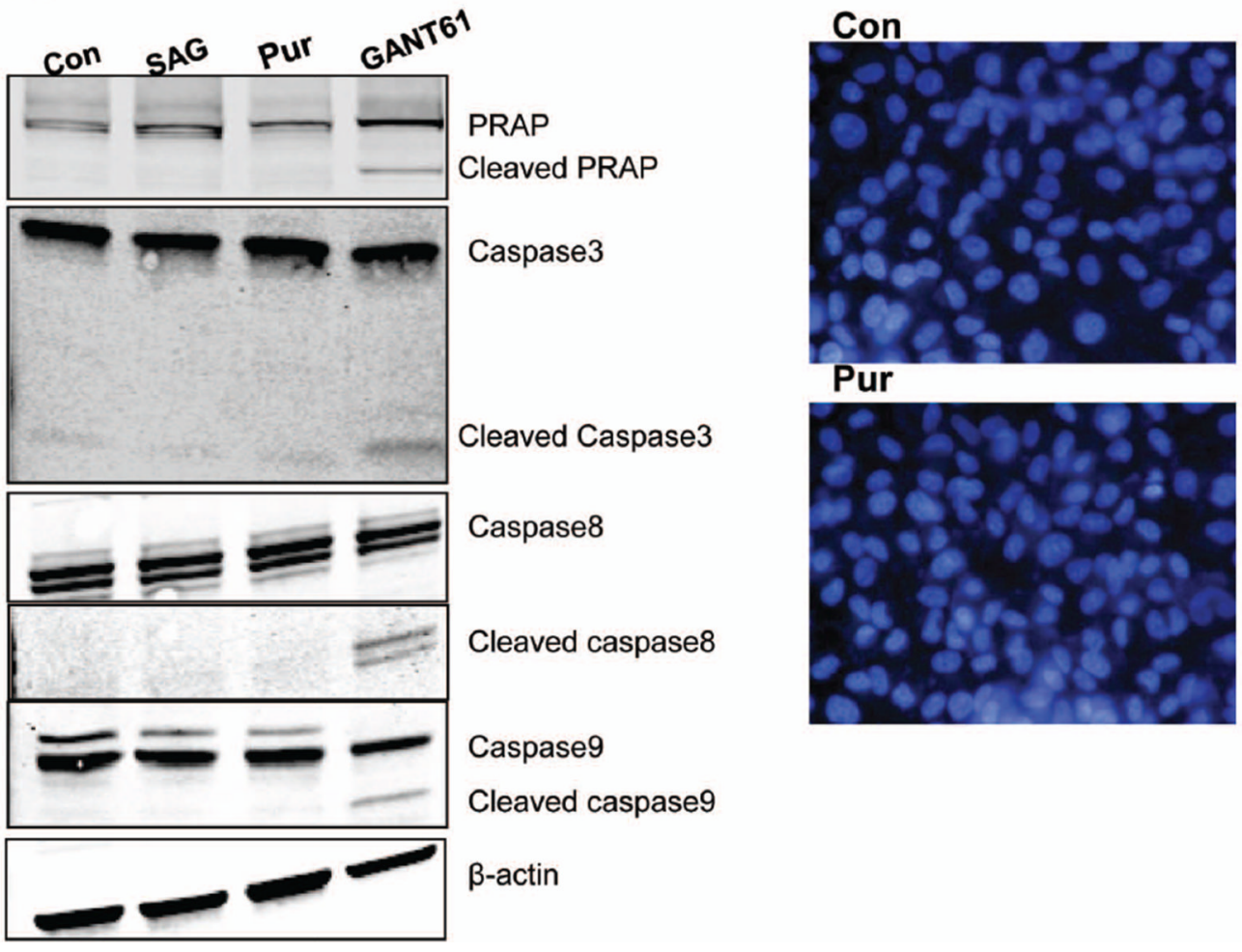

$$
\text { Pur }
$$

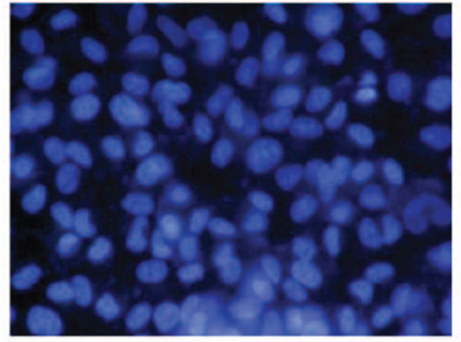

SAG

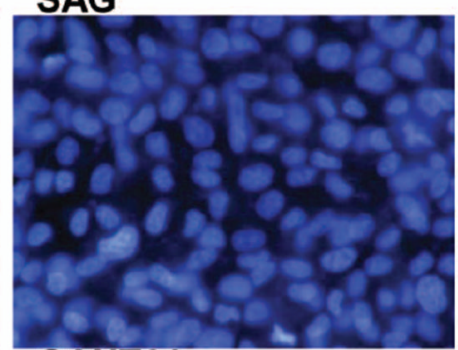

GANT61

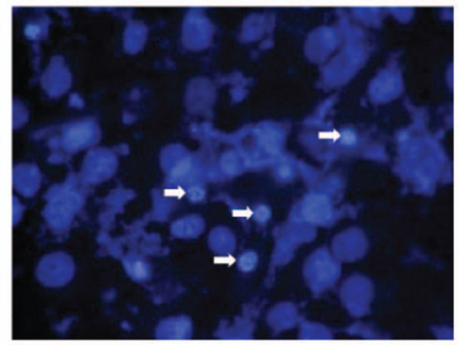

B
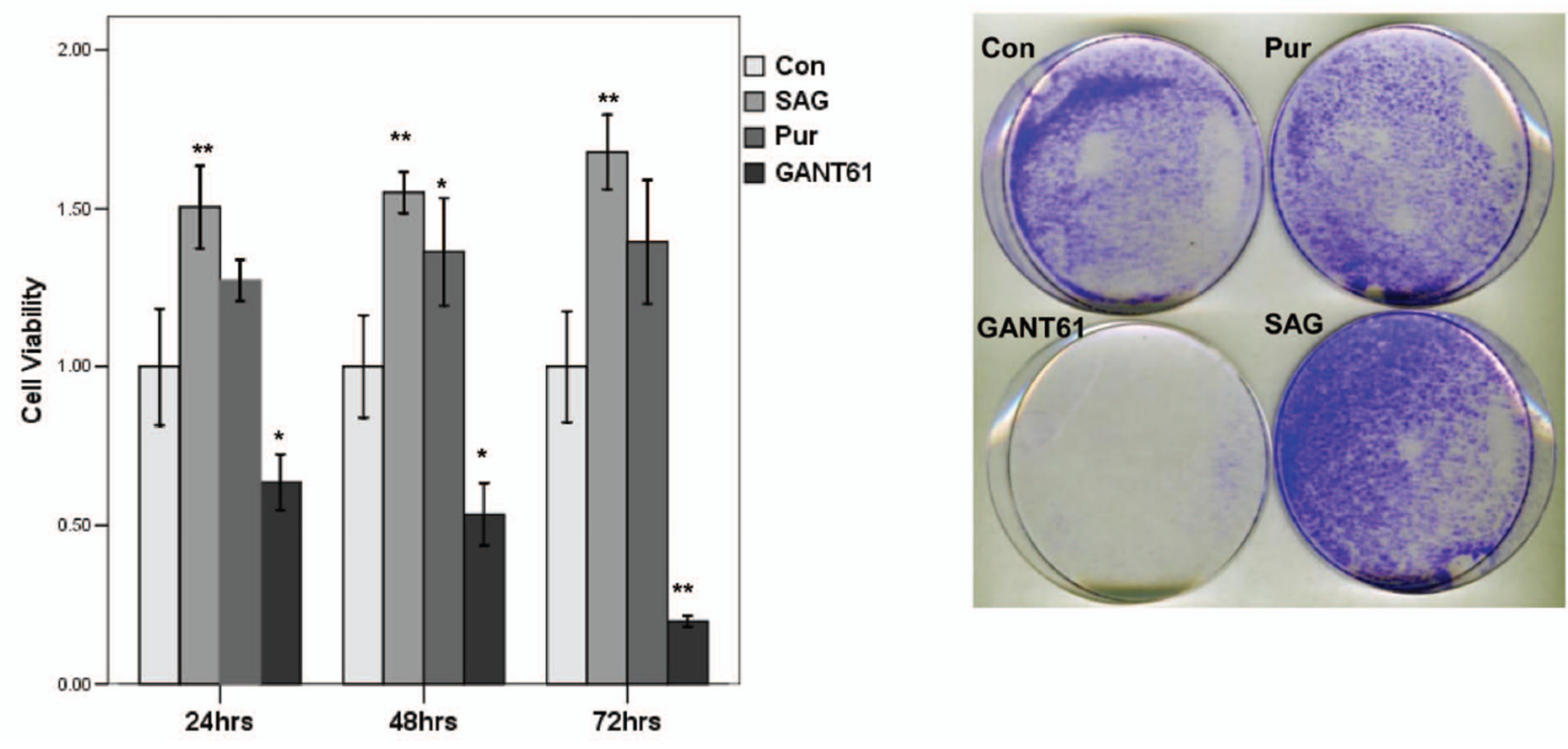
C
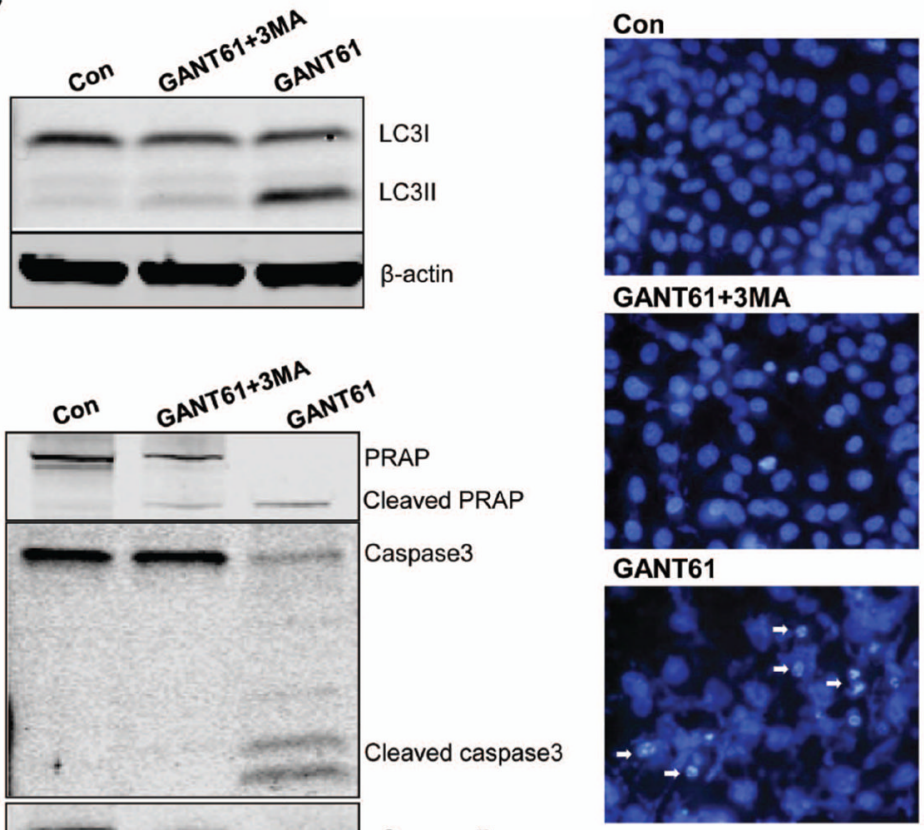

Con

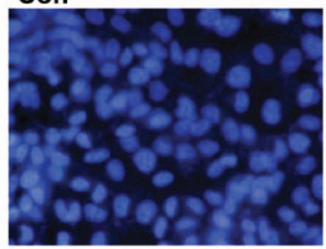

GANT61+3MA

GANT61

Caspase7

Caspase9

Cleaved caspase 9

Caspase 8

Cleaved caspase 8

$\beta$-actin

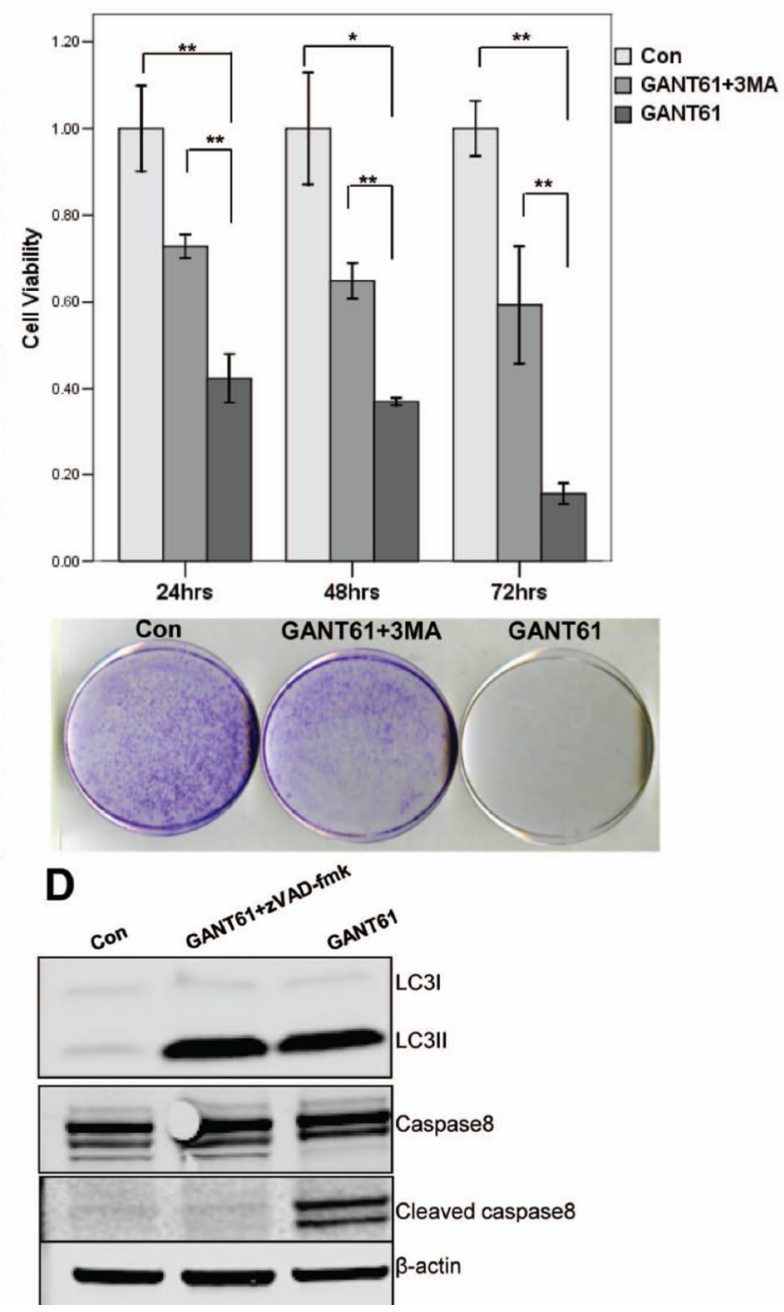


E

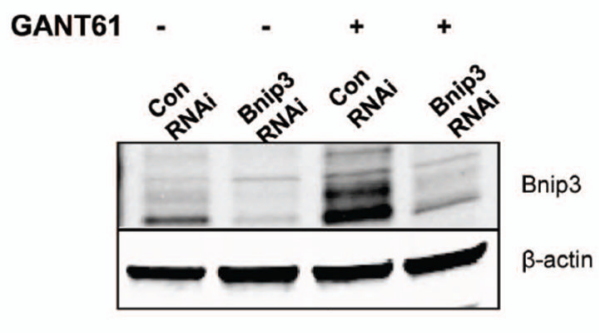

GANT61

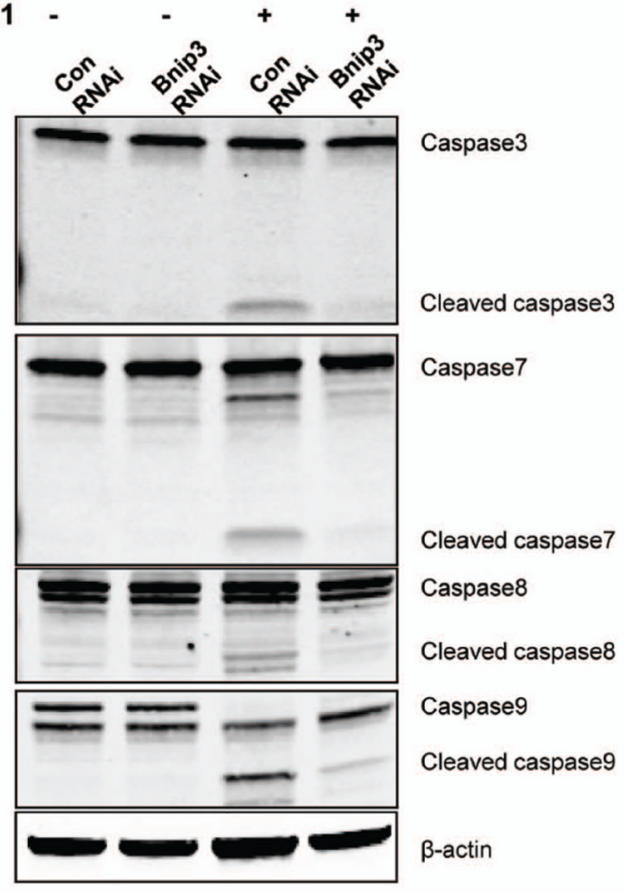

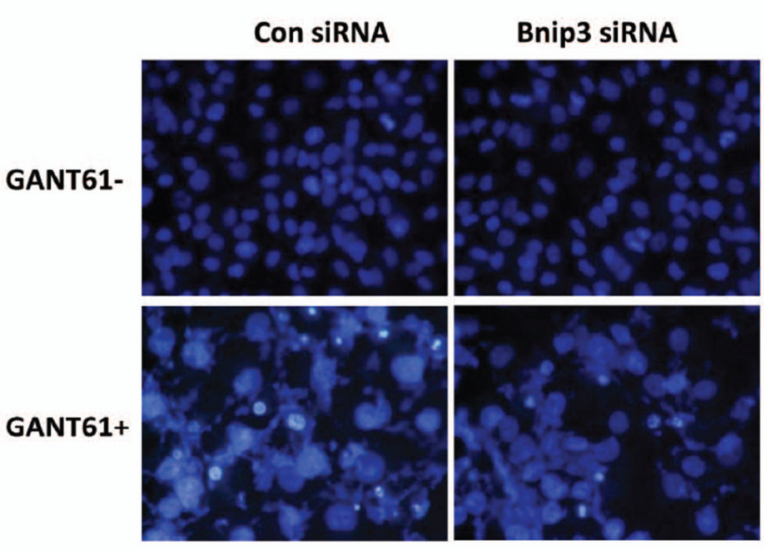

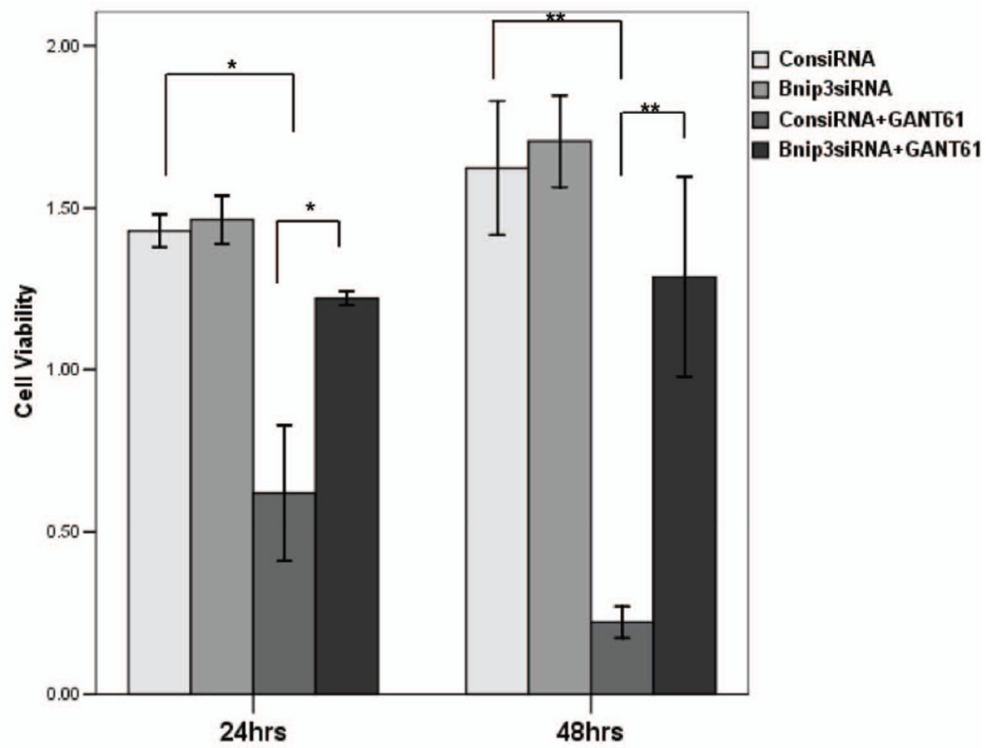



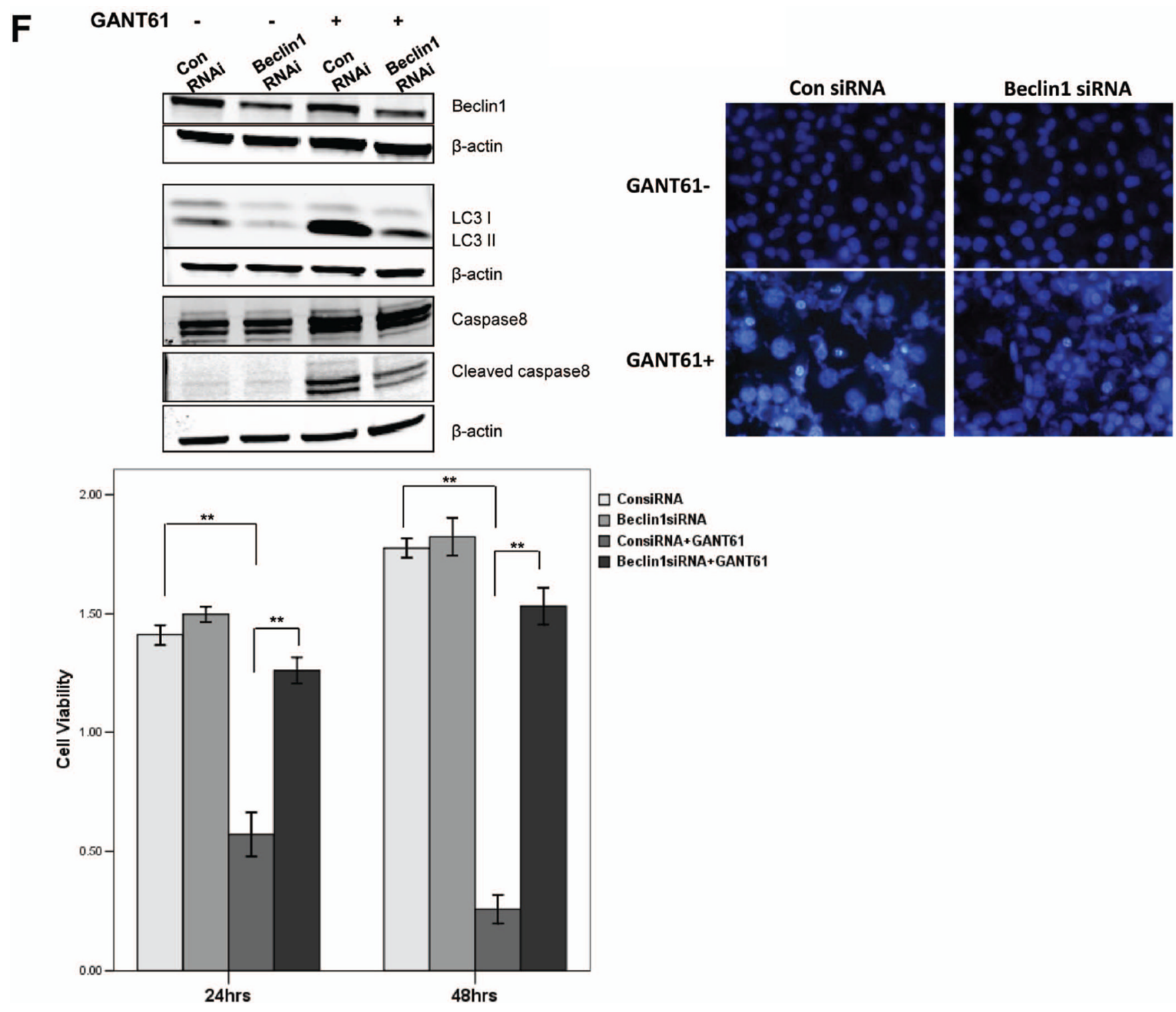

Figure 6. GANT61-induced autophagy contributes to induction of apoptosis

(A) Cell apoptosis analysis. Huh7 cells were treated for 48 hours with $0.5 \mu \mathrm{M}$ SAG, $10 \mu \mathrm{M}$ Pur, $0.4 \mu \mathrm{g} / \mathrm{ml}$ Shh or $20 \mu \mathrm{M}$ GANT61, respectively. (Left) Western blotting for PARP, caspase 3, 8, 9. (Right) Hoechst 33342 staining (arrows indicate apoptotic nuclei characterized by chromatin hypercondensation and/or nuclear fragmentation). (B) Cell viability and colonogenic assays (Huh7). (Left panel) WST-1 assay for cell viability ( $\mathrm{n}=3$; $* * p<0.01, * p<0.05$ compared to vehicle control). (Right panel) Representative colonogenic assay. (C) Huh7 cells were treated with GANT61 in the absence or presence of 3-MA and the cells were analyzed for apoptosis, viability $\left(* * p<0.01,{ }^{*} p<0.05\right)$ and colonogenic assays. Successful inhibition of autophagy by 3-MA was confirmed by LC3I/II western blotting (upper left panel). (D) Western blotting for caspase-8 and LC3I/II in Huh7 cells treated with $20 \mu \mathrm{M}$ GANT61 in the presence of $20 \mu \mathrm{g} / \mathrm{ml}$ zVAD-fmk. (E) Knockdown of Bnip3 by siRNA prevents GANT61-induced apoptosis and cytotoxicity. Huh7 cells transfected with Bnip3 siRNA or control siRNA were treated with GANT61 for 48 hours and the cells were analyzed for apoptosis by western blotting analysis and Hoechst 33342 staining, and for viability by WST-1 assay at indicated time points $(* * \mathrm{p}<0.01, * \mathrm{p}<0.05)$. (F) Knockdown of 
Beclin-1 by siRNA prevents GANT61-induced apoptosis and cytotoxicity. Huh7 cells transfected with Beclin-1 siRNA or control siRNA were treated with GANT61 for 48 hours and the cells were analyzed by western blotting, Hoechst 33342 staining, and WST-1 assay at indicated time points $(* * \mathrm{p}<0.01)$. 


\section{A}

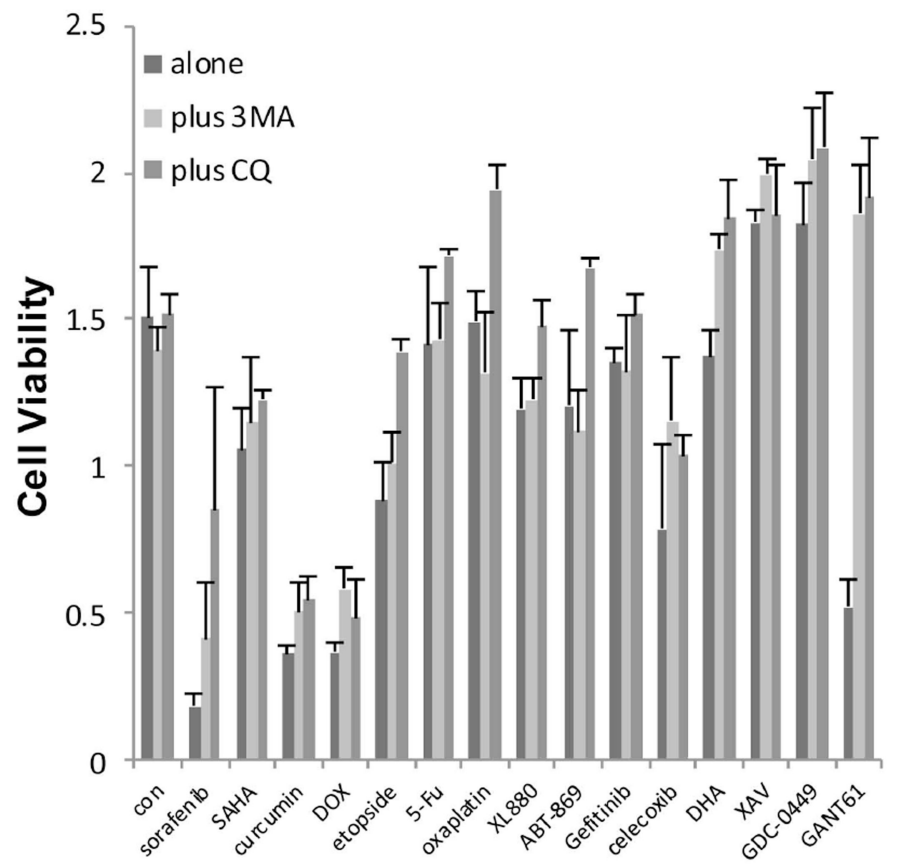

Huh7 cells

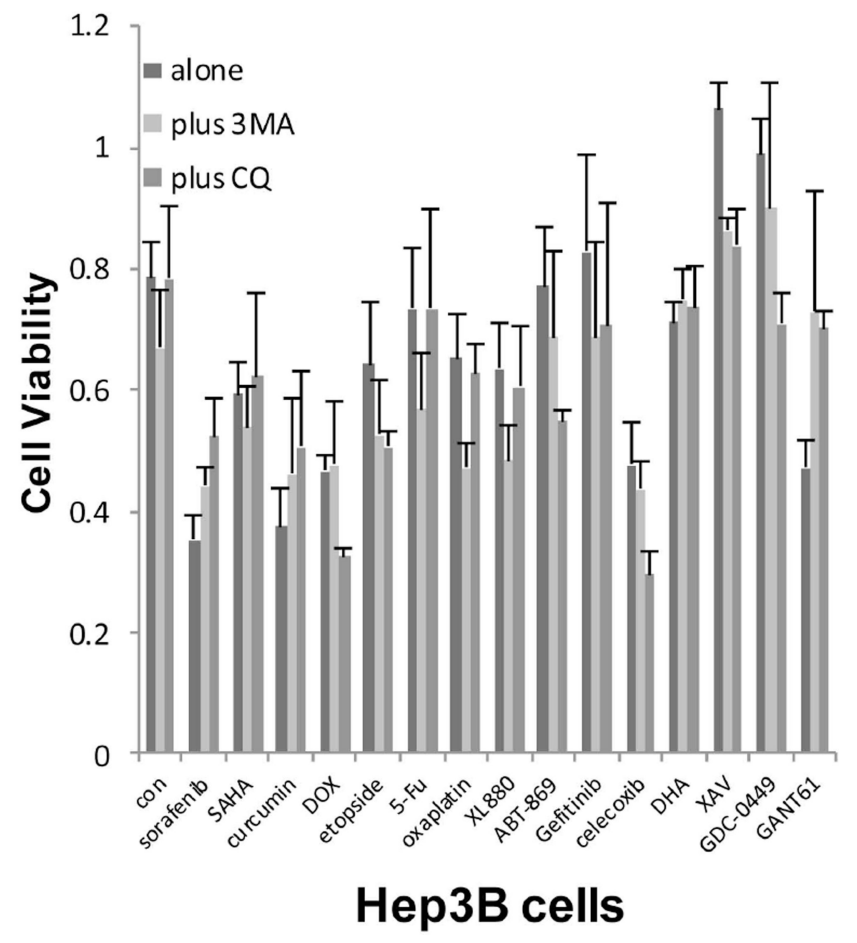




\section{A}

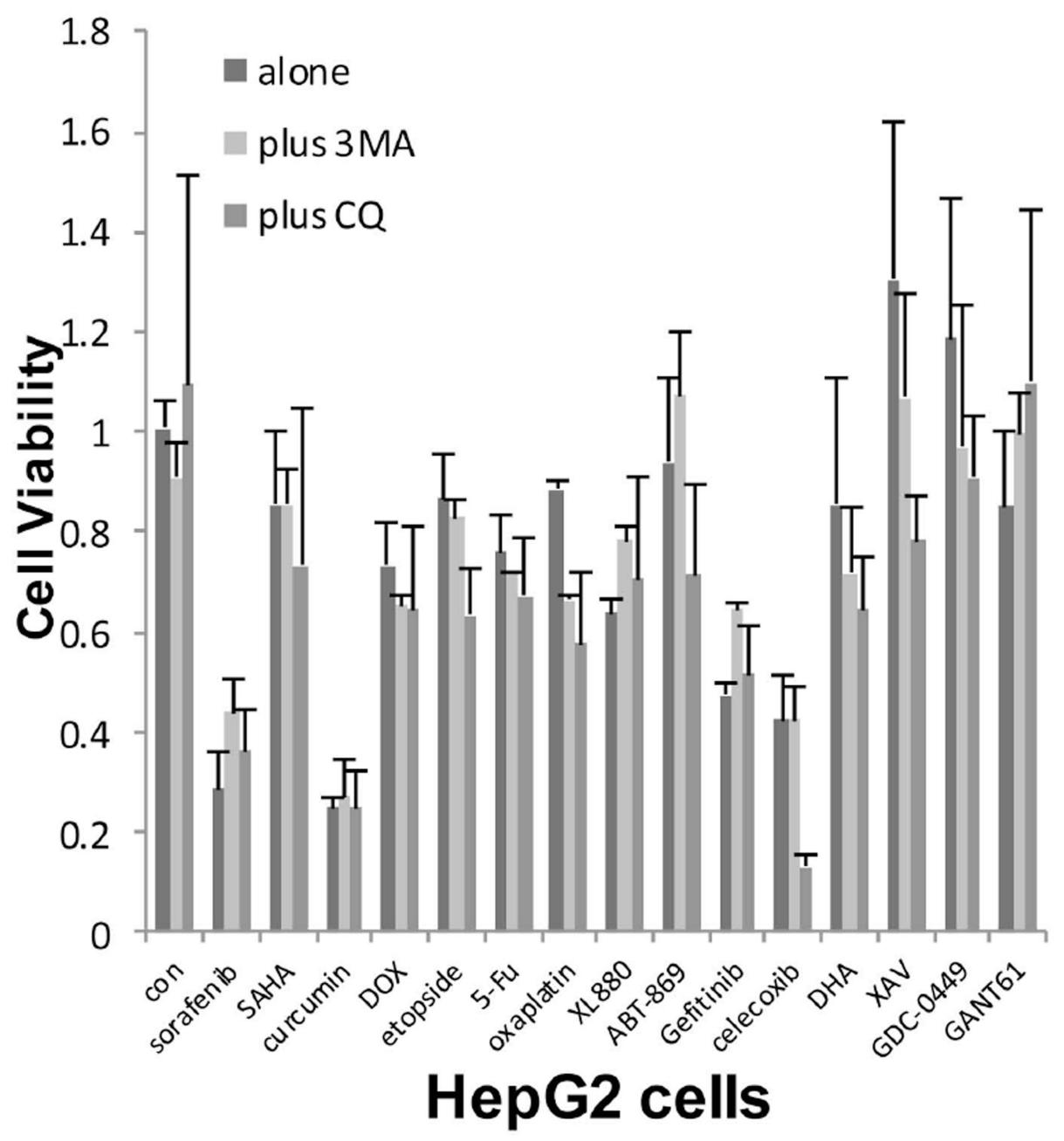


B
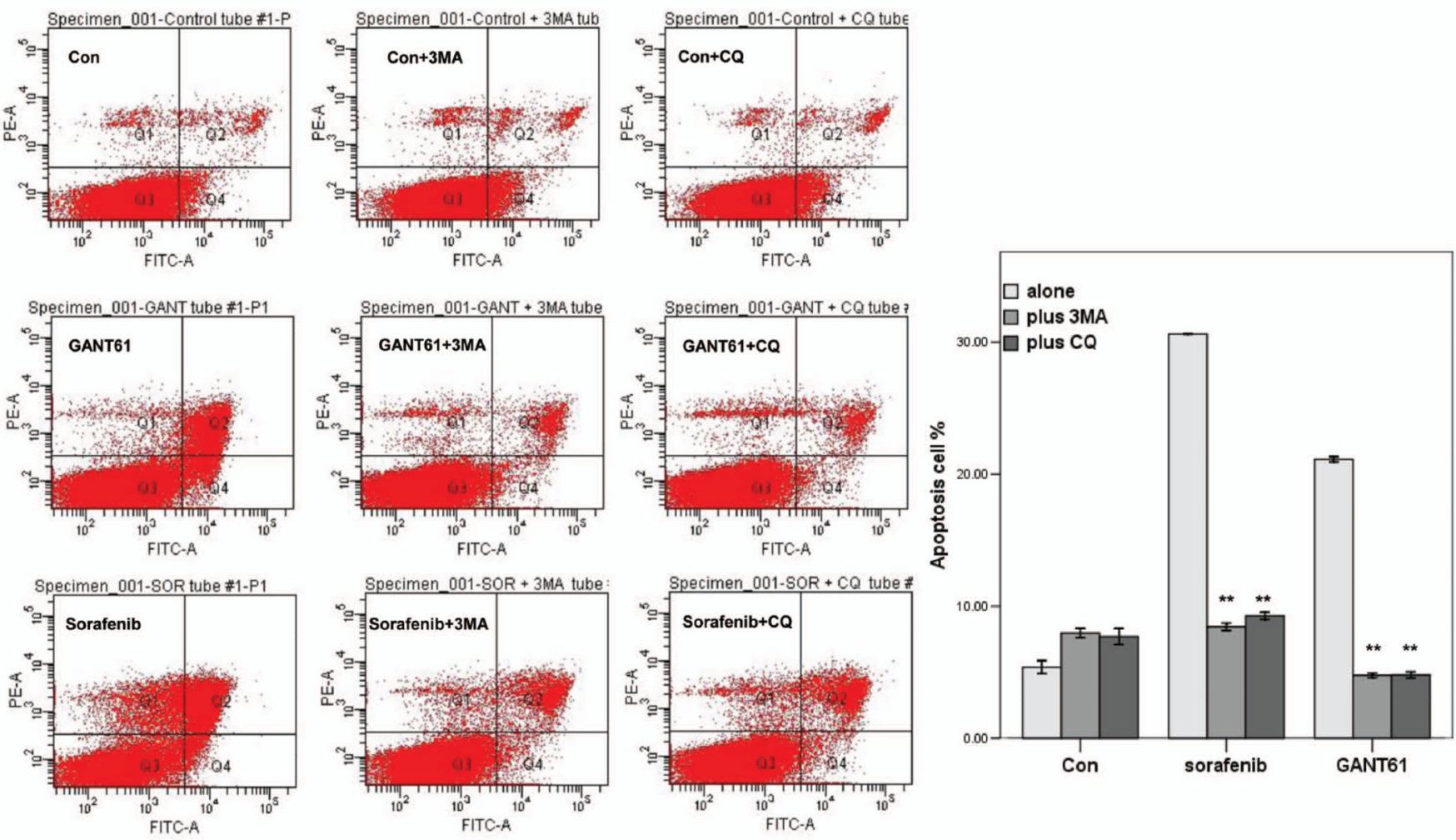

Figure 7. Inhibition of autophagy prevents GANT61-induced apoptosis

(A) Cell viability was measured by WST-1 assay in Huh7, Hep3B and HepG2 cell lines with indicated treatments for 24 hours. (B) Cell apoptosis was determined by flow cytometry using Annexin-V/propidium iodide staining in Huh7 cells treated with sorafenib or GANT61 with 3MA or CQ. The horizontal and vertical axes represent labeling with Annexin VFluorescein and PI, respectively. LR (Q4) represents early apoptotic cells (positive for Annexin V only), UR (Q2) represents late apoptotic cells (positive for both Annexin V and $\mathrm{PI})$, and LL (Q3) represents live cells. ${ }^{* *} \mathrm{p}<0.01$ compared to sorafenib or GANT61 treatment alone. 
A

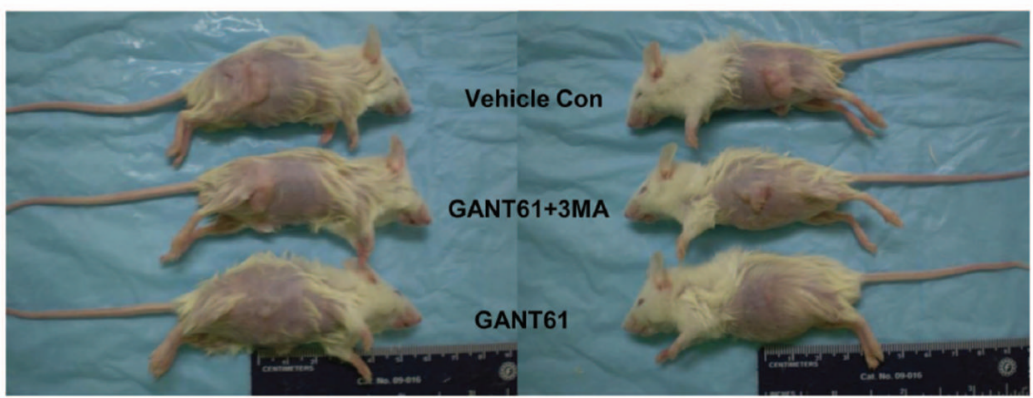

C
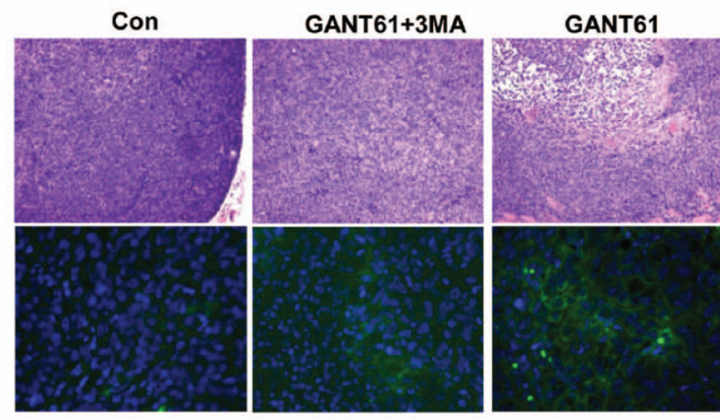

H\&E staining

D

B
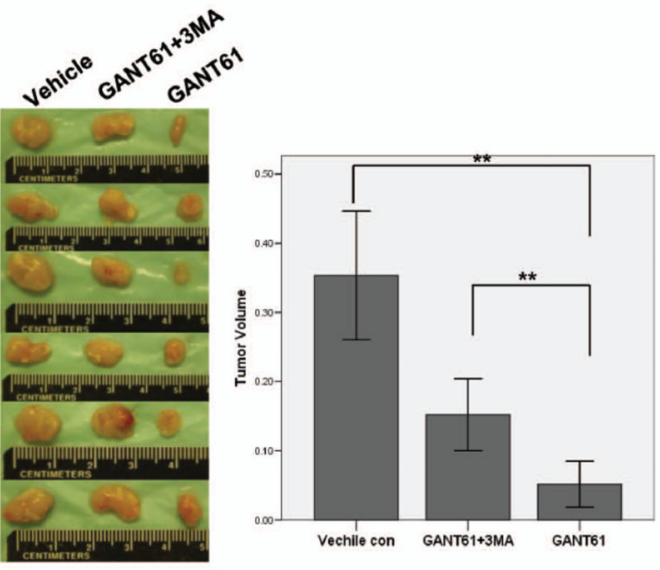

Vehicle Con GANT61+3MA

GANT61
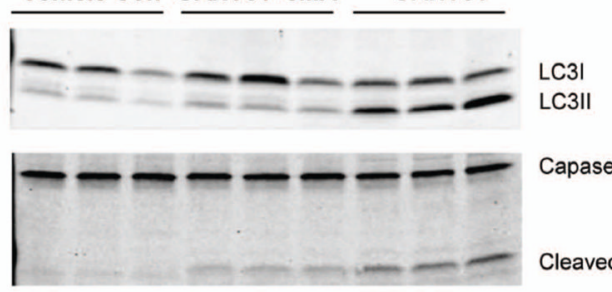

Capase3

Cleaved caspase 3

LC3II staining

Figure 8. GANT61 induces autophagy and suppresses HCC growth in vivo

$1 \times 10^{7}$ Huh7 cells suspended in a total volume of $100 \mu$ phosphate buffered saline (PBS) was subcutaneously inoculated into the flank of SCID mice. One week after inoculation, the mice were randomized to three groups and treated with vehicle control, GANT61 (50 mg/ $\mathrm{kg})$, or GANT61 (50 mg/kg) plus 3-MA $(10 \mathrm{mg} / \mathrm{kg})$ via intraperitoneal injection (i.p.) every other day for 4 weeks. (A) Photography of xenograft tumor in SCID mice. (B) The volume of xenograft tumors. Data represent mean \pm SD from 6 xenograft tumors in each group (**p<0.01). (C) H\&E staining (upper panel) and LC3II immunofluorescence staining (lower panel) of the xenograft tumor tissues. (D) Representative Western blots for LC3II, caspase-3 and caspase- 8 in xenograft tumor tissues. 